\title{
Le Collège de France : quelques données sur son histoire et son caractère propre
}

\section{OpenEdition}

\section{Journals}

Édition électronique

URL : https://journals.openedition.org/annuaire-cdf/16547

DOI : $10.4000 /$ annuaire-cdf. 16547

ISBN : 978-2-7226-0572-5

ISSN : 2109-9227

Éditeur

Collège de France

\section{Édition imprimée}

Date de publication : 30 décembre 2020

Pagination : 787-818

ISBN : 978-2-7226-0516-9

ISSN : 0069-5580

\section{Référence électronique}

"Le Collège de France : quelques données sur son histoire et son caractère propre ", L'annuaire du Collège de France [En ligne], 118 | 2020, mis en ligne le 01 avril 2021, consulté le 22 août 2022. URL http://journals.openedition.org/annuaire-cdf/16547; DOI : https://doi.org/10.4000/annuaire-cdf. 16547 


\section{LE COLLÈGE DE FRANCE : QUELQUES DONNÉES SUR SON HISTOIRE ET SON CARACTĖRE PROPRE}

\section{HISTOIRE DE L'INSTITUTION}

Pour une présentation succincte de l'histoire du Collège de France, on pourra se reporter aux précédents volumes de l'Annuaire du Collège de France (par exemple : Annuaire du Collège de France 2014-2015, Paris, Collège de France, 2016, p. 5-7 1). Des développements plus importants figurent dans les publications suivantes: Abel LEFrAnC, Histoire du Collège de France, Paris, 1893 ; Le Collège de France, livre jubilaire publié en 1932, avec vingt-cinq notices consacrées à l'histoire de l'enseignement donné au Collège de France ; Marcel BATAILLON, «Le Collège de France »; Revue de l'enseignement supérieur, $\mathrm{n}^{\circ} 2$ 2, 1962, p. 1-50 ; Yves LAPORTE, Le Collège de France, Paris, Collège de France, 1990 ; Christophe CHARLE et Éva TElKÈs, Les Professeurs du Collège de France. Dictionnaire biographique 1901-1939, Paris, Institut national de recherche pédagogique / Éditions du CNRS, 1988 ; Christophe ChARle, «Le Collège de France », in Pierre Nora (dir.), Les Lieux de mémoire, II : La Nation, t. 3, Paris, Gallimard, 1986, p. 389-424 ; Marc FuMAROLI (dir.), Les Origines du Collège de France (1500-1560), Paris, Klincksieck, 1998 ; André TUILIER (dir.), Histoire du Collège de France : I. La Création 1530-1560, Paris, Fayard, 2006 (préface de Marc Fumaroli). Un nouvel ouvrage est publié en 2015, doté d'une riche iconographie, pour une grande part inédite, provenant des archives de l'institution : Antoine COMPAGNON, Pierre CORVOL et John SCHEID (dir.) (avec la collaboration de Céline SURPRENANT), Le Collège de France, cinq siècles de recherche libre, Gallimard / Collège de France, 2015. On notera enfin la parution, en 2017, dans la nouvelle collection coéditée par le Collège de France et Les Belles Lettres («Docet omnia »), de l'ouvrage La Politique des chaires au Collège de France, sous la direction de Wolf FEUERHAHN ${ }^{2}$, publié dans le cadre du projet de recherche « Passage des disciplines : histoire globale du Collège de France XIX ${ }^{\mathrm{e}}-\mathrm{XX}^{\mathrm{e}}$ siècles », dirigé par Antoine COMPAGNON.

1. En ligne : journals.openedition.org/annuaire-cdf/12705.

2. En ligne : books.openedition.org/lesbelleslettres/120. 
Les ChaIRES DU Collège DE FRANCE DEPUIS LE XIXe SIÈCLE

\section{Antiquités grecque et romaine}

Dans la chaire Langue et littérature grecques où s'étaient illustrés Jean-Baptiste GAIL (1791-1829) et Jean-François BoISSONADE (1829-1855), Jean-Pierre RosSIGNOL (1855-1892) eut comme successeurs Maurice CROISET (1893-1930) et Émile BOURGUET (1932-1938). Une chaire Épigraphie et antiquités grecques a été instituée en 1877, et occupée successivement par Paul FOUCART (1877-1926), Maurice HOLLEAUX (1927-1932) et Louis ROBERT (1939-1974). Une chaire dénommée La Grèce et la formation de la pensée morale et politique a été créée en 1973 pour Jacqueline DE ROMILLY, première femme à occuper une chaire au Collège (19731984), puis transformée, en 1984, en chaire Tradition et critique des textes grecs pour Jean IRIGOIN (1986-1992). Gabriel MILLET, qui occupa de 1926 à 1937 la chaire Esthétique et histoire de l'art, l'orienta vers l'étude du byzantinisme, auquel fut consacrée une chaire Archéologie paléo-chrétienne et byzantine pour André GRABAR (1946-1966) puis une chaire Histoire et civilisation de Byzance pour Paul LEMERLE (1967-1973) ; elle est devenue Histoire et civilisation du monde byzantin pour Gilbert DAGRON de 1975 à 2001. En 1992, a été créée une chaire Histoire économique et monétaire de l'Orient hellénistique pour Georges LE RIDER (1993-1998); en 2002, une chaire Épigraphie et histoire des cités grecques occupée par Denis KNOEPFLER (2003-2014). En 2014 a été créée une chaire Culture écrite de l'Antiquité tardive et papyrologie byzantine, confiée en 2015 à Jean-Luc FOURNET, et en 2016 une chaire Religion, histoire et société dans le monde grec antique, occupée par Vinciane PIRENNE-DELFORGE en 2017.

Une chaire Philosophie grecque et latine eut comme titulaires Édouard BOSQUILLON (1775-1814), Jean-François THUROT (1814-1832), Théodore JOUFFrOY (1832-1837), Jules BARTHÉLÉMY SAINT-HILAIRE (1838-1852), Émile SAISSET (chargé de cours 1853-1857), Charles LÉVÊQUE (chargé de cours 1857-1860, titulaire 1861-1900), Henri BERGSON (1900-1904). Une chaire Histoire de la pensée hellénistique et romaine a été créée en 1981 pour Pierre HADOT (1982-1991).

Pour l'enseignement du latin, deux chaires existaient depuis l'Ancien Régime : Éloquence latine, tenue successivement par Pierre GUEROULT (1809-1816), Jean-Louis BURNOUF (1817-1844), Désiré NISARD (1844-1852), Wilhelm RINN (1853-1854), Ernest HaVET (1854-1885), et Poésie latine, par Jacques DeliLle (1778-1813), Pierre-François TISSOT (1813-1821), Joseph NAUDET (1821-1830), Pierre-François TISSOT à nouveau (1830-1854), Charles-Augustin DE SAINTEBEUVE (1854-1869) qui, toutefois, ne put jamais enseigner. La prédominance de curiosités et de méthodes nouvelles se manifesta par la transformation de la chaire Éloquence latine en Philologie latine, avec pour titulaire Louis HAVET (1885-1925), et de Poésie latine en Histoire de la littérature latine, occupée par Gaston BoISSIER (1869-1906) et Paul MoNCEAUX (1907-1934). Ces enseignements ont été complétés par ceux de disciplines qui attestaient l'élargissement de l'horizon scientifique ; une chaire Épigraphie et antiquités romaines, créée en 1861, pour Léon RENIER (1861-1885) a été occupée par Ernest DESJARDINS (1886), et ensuite par René CAGNAT (1887-1930), puis, sous le titre élargi de Civilisation romaine, par Eugène AlBertini (1932-1941), André Piganiol (1942-1954), et Jean GaGÉ (1955-1972) ; elle est devenue, de 1975 à 1999, Histoire de Rome pour Paul VEYNE. Une chaire Histoire de l'Afrique du Nord, où furent spécialement étudiées les périodes romaine 
et post-romaine, a eu pour titulaire Stéphane GSELL (1912-1932). Une chaire Histoire de la langue latine, occupée par Alfred ERNOUT (1944-1951), a été transformée en 1951 en Littérature latine pour Pierre COURCELLE (1951-1980). En 2000, une chaire intitulée Religion, institutions et société de la Rome antique a été créée pour John SCHEID (2001-2016). En 2017 est créée une chaire Droit, culture et société de la Rome antique, confiée à Dario MANTOVANI en 2018.

Une chaire Numismatique de l'Antiquité et du Moyen Âge, inaugurée en 1908 par Ernest BABELON, a été transformée, en 1924, en Numismatique de l'Antiquité, que Théodore REINACH a occupée jusqu'en 1928.

\section{Philosophie}

Un enseignement Philosophie moderne créé en 1874 a été assuré par Jean NOURRISSON (1874-1899), Gabriel TARDE (1900-1904), Henri BERGSON (1904-1921), Édouard Le RoY (1921-1940), Louis LAVELLE (1941-1951), Maurice MERLEAU-PONTY (1952-1961). En 1962, fut créée une chaire Philosophie de la connaissance pour Jules VUILLEMIN qui l'occupa jusqu'en 1990. Une chaire Histoire de la philosophie au Moyen Âge, tenue de 1932 à 1950 par Étienne GILSON, a été remplacée par une chaire Histoire et technologie des systèmes philosophiques pour Martial GuÉROULT (1951-1962), et dénommée ensuite Histoire de la pensée philosophique pour Jean HYPPOLITE (1963-1968). Cette chaire a été transformée en Histoire des systèmes de pensée pour Michel FouCAULT (1970-1984), puis, de 1985 à 1990, en Épistémologie comparative pour Gilles-Gaston GRANGER. Une chaire Philosophie du langage et de la connaissance, créée en 1994, est occupée de 1995 à 2010 par Jacques BOUVERESSE. Lui succède une chaire Métaphysique et philosophie de la connaissance, confiée en 2010 à Claudine TIERCELIN. En 2012 est créée une chaire Philosophie médiévale, confiée en 2013 à Alain DE LIBERA.

En 1999, ont été créées une chaire Philosophie des sciences biologiques et médicales pour Anne FAgOT-LARgEAult (2000-2009) et une chaire Philosophie et histoire des concepts scientifiques pour Ian HACKING (2001-2006).

\section{Linguistique générale}

L'enseignement Grammaire comparée fut inauguré au Collège de France par Michel BRÉAL (1866-1905), qui eut pour successeurs Antoine MEILLET (1906-1936) et Émile BenVENISTE (1937-1972). Pendant un temps s'y trouva rattaché un Laboratoire de phonétique expérimentale, dont le premier directeur fut l'abbé ROUSSELOT ; une chaire Phonétique expérimentale fut occupée par ce savant de 1923 à 1924. Une chaire Théorie linguistique est créée en 1986 pour Claude HAGÈGE (1988-2006).

\section{Langues, histoire et littératures occidentales}

L'enseignement Littérature française fut d'abord représenté par Antoine DE Cournand (1784-1814) et Stanislas ANDRIEuX (1814-1833). Ce fut le début d'une tradition à laquelle se rattachent les noms de Jean-Jacques AMPÈRE (18331864), de Louis DE LOMÉNIE (1864-1878), de Paul ALBERT (1878-1880), d'Émile DESCHANEL (1881-1903), d'Abel LEFRANC (1904-1937). La chaire a été transformée en Poétique, occupée par Paul VALÉRY (1937-1945), puis en Histoire des créations 
littéraires en France pour Jean POMMIER (1946-1964). Cette chaire a été ensuite consacrée à l'enseignement de la Littérature française moderne ; elle a été occupée par Georges BLIN de 1965 à 1988. Une chaire Sémiologie littéraire a été créée pour Roland BARTHES (1976-1980). En 1980 a été créée pour Yves BONNEFOY une chaire Études comparées de la fonction poétique (1981-1993). En 1986, a été créée une chaire intitulée Rhétorique et société en Europe (XVI $\mathrm{e}-\mathrm{XVII}{ }^{\mathrm{e}}$ siècles) pour Marc FUMAROLI (1987-2002); et une chaire Littératures modernes de l'Europe néolatine a été créée pour Carlo Ossola en 1998. En 2005 sont créées une chaire Littérature française moderne et contemporaine : histoire, critique, théorie, occupée à partir de 2006 par Antoine COMPAGNON, et une chaire intitulée Écrit et culture dans l'Europe moderne, occupée de 2006 à 2016 par Roger CHARTIER.

Une chaire spécialement consacrée à Langue et littérature françaises du Moyen Âge, a été inaugurée par Paulin PARIS (1853-1872), qui eut pour successeurs Gaston PARIS (1872-1903), puis Joseph BÉDIER (1903-1936). Remplacée ensuite par une chaire Histoire du vocabulaire français occupée par Mario RoQUES (1937-1946), elle a été rétablie sous son ancien titre pour Félix LECOY, de 1947 à 1974, puis en 1993 sous le titre de Littératures de la France médiévale pour Michel ZINK (1995-2016).

En 1905, une chaire Histoire et antiquités nationales s'y ajouta pour Camille JULLIAN (1905-1930), tenue ensuite par Albert GRENIER (1936-1948).

En 1964, a été créée une chaire Archéologie et histoire de la Gaule pour PaulMarie Duval (1964-1982), transformée en 1983 en chaire Antiquités nationales pour Christian Goudineau (1984-2010). En 2011, une chaire Techniques et économies de la Méditerranée antique a été créée pour Jean-Pierre BRUN.

D'autre part, deux chaires nouvelles, instituées en 1925 et en 1932, concernaient l'Europe médiévale : l'une, occupée par Edmond FARAL (1925-1954), s'intitulait Littérature latine du Moyen Âge, l'autre déjà mentionnée en philosophie, fut occupée par Étienne GILSON, de 1932 à 1950. En 1969 a été créée une chaire Histoire des sociétés médiévales pour Georges DuBY, devenue en 1991 Histoire de l'Occident méditerranéen au Moyen Âge pour Pierre TOUBERT, qui l'a occupée de 1992 à 2003. En 2014 est créée une chaire Histoire des pouvoirs en Europe occidentale, XIII $-\mathrm{XVI}^{\mathrm{e}}$ siècle, occupée à partir de 2015 par Patrick BOUCHERON.

Consacrée à l'activité extérieure de la France, une chaire, fondée par les principales colonies d'alors, a été occupée sous le titre d'Histoire coloniale par Alfred MARTINEAU (1921-1935), puis, sous le titre d'Histoire de la colonisation, par Edmond ChassigneuX (1939-1946), et ensuite, sous le titre d'Histoire de l'expansion de l'Occident, par Robert MONTAGNE (1948-1954).

Enfin a été créée, en 1984, une chaire Histoire de la France contemporaine, pour Maurice AGULHON, qui l'a occupée jusqu'en 1997.

La chaire Langues et littératures slaves fut inaugurée en 1840 par le poète polonais, chargé de cours, Adam MiCKIEWICZ (1840-1852) puis par Cyprien ROBERT (chargé de cours 1852-1857) et Alexandre CHODZKO (chargé de cours 1857-1883), et occupée plus tard par Louis LÉGER (1885-1923), André MAZON (1923-1951) et André VAILlANT (1952-1962). En 1992 a été créée une chaire Histoire moderne et contemporaine du monde russe pour François-Xavier COQUIN (1993-2001).

Une chaire Langues et littératures de l'Europe méridionale, qui eut pour titulaires successifs Edgar QUINET (de 1841 à 1852 et de 1870 à 1875), Paul MEYER (18761906), Alfred MoREL-FATIO (1907-1924), a été rétablie, en 1925, sous le titre d'Histoire des littératures comparées de l'Europe méridionale et de l'Amérique latine pour Paul HAZARD (1925-1944). Depuis, deux des domaines qu'elle recouvrait 
ont été distingués par la création, en 1945 et en 1946, de deux chaires consacrées l'une à Langues et littératures de la péninsule ibérique et de l'Amérique latine, tenue par Marcel BATAILLON (1945-1965), puis par Israël RÉVAH (1966-1973) ; l'autre à l'Histoire de la civilisation italienne pour Augustin RENAUDET de 1946 à 1950, transformée Langues et civilisation italiennes pour André PÉZARD de 1951 à 1963. En 1992, une chaire Langues et littératures romanes a été créée pour Harald WEINRICH, qui l'occupa jusqu'en 1998.

Une chaire Langues et littératures d'origine germanique eut pour premiers titulaires Philarète CHASLES (1841-1873) et Guillaume GuIZOT (1874-1892). Celui-ci fut suppléé par Jean-Jules JUSSERAND, puis par Arthur CHUQUET, qui devint titulaire de la chaire en 1893 et l'occupa jusqu'en 1925. Lui succédèrent Charles ANDLER (1926-1933), Ernest ToNNELAT (1934-1948), Fernand Mossé (1949-1956), et Robert MiNDER de 1957 à 1973. En 1984 a été créée une chaire Grammaire et pensée allemandes, pour Jean-Marie ZEMB, qui l'occupa jusqu'en 1998.

Une chaire Langues et littératures celtiques a été occupée par Henry D'ARBOIS DE JUBAINVILLE (1882-1910) puis par Joseph LOTH (1910-1930).

Une chaire Civilisation américaine, créée en 1931, pour Bernard FAŸ (révoqué en 1945), a été transformée pour Marcel GIRAUD, de 1947 à 1971, en Histoire de la civilisation de l'Amérique du Nord.

Une chaire Étude de la création littéraire en langue anglaise a été créée en 2001 pour Michael EDWARDS (2003-2008).

\section{Langues, histoires et littératures orientales}

L'enseignement de l'hébreu, le plus ancien de tous, donné par Étienne QUATREMÈRE (1819-1857), puis par Louis DUBEUX (chargé de cours 1857-1861), a été illustré ensuite par Ernest RENAN (1862-1864 et 1870-1892), par Salomon MUNK (1864-1867) et par Philippe BERGER (1893-1910). Celui de l'araméen a été assuré par Rubens DuVAL (1895-1907). Après un long intervalle, une chaire intitulée Hébreu et araméen a été instituée de 1963 à 1971 pour André DUPONT-SOMMER ; puis pour André CAQUOT de 1972 à 1994. Une chaire Épigraphie et antiquités sémitiques a été créée pour Charles CLERMONT-GANNEAU (1890-1923), une autre, Histoire ancienne de l'Orient sémitique, a été occupée par Isidore LÉVY (1932-1941) et de 1995 à 2001, une chaire Antiquités sémitiques occupée par Javier TEIXIDOR.

L'égyptologie a fait son entrée au Collège avec son fondateur, Jean-François CHAMPOLLION (1831-1832), dans une chaire Archéologie tenue ensuite par Jean-Antoine LETRONNE (1837-1848), puis par Charles LENORMANT (1849-1859). Elle devint Philologie et archéologie égyptiennes avec Emmanuel DE RougÉ (18601872) et Gaston MASPERO (1874-1916), fut reprise par Alexandre MORET (19231938), et occupée successivement par Pierre LACAU (1938-1947), Pierre MONTET (1948-1956), Étienne DrIOTON (1957-1960), et Georges POSENER (1961-1978). Elle a subsisté sous le titre d'Égyptologie pour Jean LECLANT (1979-1990), puis pour Jean YOYOTTE (1991-1997). En 1999, une chaire Civilisation pharaonique : archéologie, philologie, histoire a été créée pour Nicolas GRIMAL.

L'enseignement de l'assyriologie a été ouvert aussi par un fondateur, Jules OPPERT (1874-1905), dans une chaire Philologie et archéologie assyriennes où lui a succédé Charles FoSSEY (1906-1939). Après un intervalle, il a été repris par Édouard DHORME (1945-1951) sous le titre de Philologie et archéologie assyro-babyloniennes et poursuivi sous celui d'Assyriologie par René LABAT de 1952 à 1974, par Paul 
GARELli, de 1986 à 1995 et par Jean-Marie DuRAND, de 1999 à 2011. Une chaire Civilisation mésopotamienne a été créée pour Dominique CHARPIN en 2013.

Une chaire Archéologie de l'Asie occidentale a été créée en 1953 pour Claude SCHAEFFER-FORRER qui l'a occupée jusqu'en 1969. Enfin, en 1973 était créée une chaire Langues et civilisation de l'Asie Mineure pour Emmanuel LAROCHE, qui l'a occupée jusqu'en 1985.

Une chaire Histoire et civilisation du monde achéménide et de l'empire d'Alexandre a été créée en 1998 pour Pierre BRIANT, qui l'occupe de 1999 à 2012.

La chaire Arabe a été tenue successivement par Antoine CAUSSIN DE PERCEVAL (1783-1833), Armand-Pierre CAUSSIN DE PERCEVAL (1833-1871), Charles-François DEFRÉMERY (1871-1883), Stanislas GUYARD (1884), Adrien BARBIER DE MEYNARD (1885-1908), Paul CASANOVA (1909-1926), William MARÇAIS (1927-1943). Elle a été transformée en Histoire du monde arabe pour Jean SAUVAGET (1946-1950). Devenue Langue et littérature arabes, elle a été occupée par Gaston WIET (19511959). À côté d'elle furent fondées : en 1902, une chaire Sociologie et sociographie musulmanes, inaugurée par Alfred LE CHATELIER (1902-1925), occupée ensuite par Louis MASSIGNON (1926-1954), modifiée en Sociologie musulmane pour Henri LAOUST de 1956 à 1975, et transformée en 1976 en Langue et littérature arabes classiques pour André MiQUEL, occupée jusqu'en 1997 - puis en 1941, une chaire Histoire des arts de l'Orient musulman pour Albert GABRIEL (1941-1953). En 1956 était créée une chaire Histoire sociale de l'Islam contemporain, occupée par Jacques BERQUE jusqu'en 1981. Une chaire Histoire contemporaine du monde arabe est créée en 2003 pour Henry LAURENS.

Les chaires Turc et de Persan ont été réunies de 1784 à 1805 par Pierre RUFFIN, qui abandonna à partir de 1805 le persan au plus illustre islamisant de l'époque, Isaac SILVESTRE DE SACY (1806-1838). Lui succédèrent: Amédée JAUBERT (1838-1847), Jules MOHL (1850-1876), Adrien BARBIER DE MEYNARD (1876-1885), James DARMESTETER (1885-1894). Après Pierre RUFFIN (1805-1822) l'enseignement du turc seul a été assuré par Daniel KIEFFER (1822-1833), Alix DESGRANGES (1833-1854), Mathurin-Joseph COR (1854), Abel PAVET DE COURTEILLE (chargé de cours 1854-1861, titulaire 1861-1889). En 1997, a été créée une chaire Histoire turque et ottomane pour Gilles VeINSTEIN, qui l'a occupée de 1999 à 2012.

Les domaines de recherche nouveaux entrés dans l'enseignement du Collège au XIX ${ }^{\mathrm{e}}$ siècle ont d'abord été ceux de l'Inde et de la Chine, dont l'étude avait été amorcée en Europe par plusieurs orientalistes du Collège au siècle précédent. En 1814, furent créées ensemble les chaires Sanscrit et Chinois.

La première a été inaugurée par Léonard DE CHÉZY (1814-1832), illustrée par Eugène BURNOUF (1932-1852), et reprise après un intervalle de suppléances par Édouard FouCAUX (1862-1894), puis Sylvain LÉVI (1894-1935) et Jules BLOCH (1937-1951). L'enseignement y débordant traditionnellement le domaine du sanscrit, elle a repris en 1951, la dénomination de Langues et littératures de l'Inde et a eu pour titulaire Jean FILLIOZAT de 1952 à 1978. En 1983 a été créée une chaire Histoire du monde indien pour Gérard FUSSMAN (1984-2011), et, en 1993, une chaire Langues et religions indo-iraniennes pour Jean KELLENS (1993-2014).

La seconde, dont l'enseignement s'est, de son côté, constamment étendu à l'ensemble de la sinologie, a été tenue par Jean-Pierre ABEL-RÉMUSAT (1814-1932), Stanislas JULIEN (1832-1873), Léon D’HERVEY DE SAINT-DENYS (1874-1892), Édouard Chavannes (1893-1918), Henri MASPERo (1921-1945), Paul DemiÉville 
(1946-1964), Jacques GERNET (1975-1992); depuis 1991 une chaire Histoire de la Chine moderne est occupée par Pierre-Étienne WILL (1991-2014) et une chaire Histoire intellectuelle de la Chine est confiée en 2008 à Anne CHENG.

Étendant le champ des enseignements aux pays d'influence indienne et chinoise et aux civilisations propres à ces pays, trois chaires ont été créées : la première, Langues, histoire et archéologie de l'Asie centrale pour Paul PELLIOT (1911-1945), qui devait prendre le titre d'Histoire et civilisations de l'Asie centrale pour Louis HAMBIS, de 1965 à 1977, et se transformer en Sociographie de l'Asie du Sud-Est pour Lucien BERNOT (1978-1985) ; la deuxième, Histoire et philologie indochinoises pour Louis FinOT (1920-1930), auquel ont succédé Jean PRZYLUSKI (1931-1944), puis Émile GASPARDONE, de 1946 à 1965, et qui a été alors transformée en Étude du monde chinois : institutions et concepts pour Rolf A. STEIN (1966-1981) ; enfin la troisième, sous le titre de Civilisations d'Extrême-Orient, a été occupée par Paul Mus (19461969). Une chaire Étude du bouddhisme a été créée en 1970 pour André BAREAU, qui l'a occupée jusqu'en 1991. En 1979, a été créée une chaire Civilisation japonaise pour Bernard FRANK qui l'a occupée jusqu'en 1996. Enfin, en 2011, une chaire Philologie et civilisation japonaise a été créée pour Jean-Noël ROBERT. En 2012, une chaire Histoire et cultures de l'Asie centrale pré-islamique a été créée pour Frantz GRENET qui l'occupe depuis 2013.

\section{Droit et sciences humaines}

Depuis l'Ancien Régime existait une chaire Droit de la nature et des gens, tenue au XIX ${ }^{\mathrm{e}}$ siècle par Pierre DE PASTORET (1804-1821), Xavier DE PORTETS (1822-1854), Adolphe FRANCK (1856-1887). En 1831 commençait, avec Eugène LERMINIER (1831-1849), un enseignement Histoire générale et philosophique des législations comparées, qui fut continué par Édouard LABOULAYE (1849-1883), et par Jacques FLACH (1884-1919). En 1979, une chaire Droit international est créée pour RenéJean DUPUY qui l'a occupée jusqu'en 1989. Une chaire Études juridiques comparatives et internationalisation du droit a été créée en 2001 pour Mireille DELMAS-MARTY (2002-2011). En 2012, une chaire intitulée État social et mondialisation : analyse juridique des solidarités est confiée à Alain SUPIOT.

Dès 1831, était instituée pour Jean-Baptiste SAY une chaire Économie politique, qui fut occupée après lui par Pellegrino ROSSI (1833-1840), Michel CHEVALIER (1840-1879), et Paul LEROY-BEAUlieU (1880-1916). Une chaire Histoire des doctrines économiques, créée en 1871 pour Émile LEVASSEUR, fut transformée sur sa demande en 1885 en Géographie, histoire et statistiques économiques. En 1911, elle devint Étude des faits économiques et sociaux pour Marcel MARION (19121932). De 1955 à 1974 une chaire Analyse des faits économiques et sociaux a été occupée par François PERROUX ; en 1987 a été créée une chaire Analyse économique pour Edmond MALINVAUD qui l'a occupée jusqu'en 1993. Une chaire Théorie économique et organisation sociale a été créée en 1998 pour Roger GUESNERIE (2000-2013). En 2014 est créée une chaire Économie des institutions, de l'innovation et de la croissance, confiée à Philippe AGHION.

En 1958 une chaire Anthropologie sociale était créée pour Claude LÉVI-STRAUSS (1959-1982), et en 1971, une chaire Anthropologie physique pour Jacques RUFFIÉ (1972-1992). En 1981, a été créée pour Françoise HÉRITIER une chaire Étude comparée des sociétés africaines (1982-1998) et en 1992 pour Nathan WACHTEL une chaire Histoire et anthropologie des sociétés méso- et sud-américaines (1992-2005). 
En 1999, une chaire Anthropologie de la nature a été créée pour Philippe DESCOLA, qui l'occupe depuis 2000.

En 1917, un cours complémentaire Assurances sociales fondé par Alfred MAYEN, a été transformé, aux frais de la ville de Paris et du département de la Seine, en une chaire Prévoyance et assistance sociales, qu'occupa Édouard FUSTER (1917-1935).

En 1920, une chaire instituée pour dix ans, à l'initiative de la Fédération des sociétés coopératives de consommation, et affectée à l'Enseignement de la Coopération, a eu pour titulaire Charles GIDE jusqu'en 1930.

Dans le même ordre d'études, d'autres développements se sont encore produits. Une chaire Philosophie sociale, créée pour Jean IzOULET (1897-1929), a été transformée en Sociologie pour Marcel MAUSS (1931-1942), puis en Psychologie collective pour Maurice HALBWACHS (1944-1945). Une chaire Histoire et structure sociales de Paris et de la région parisienne, fondée par la ville de Paris en 1950, a été occupée par Louis CHEVAlIER (1952-1981). Une chaire Histoire du travail, fondée en 1907 par la Ville de Paris, a été occupée par Georges RENARD (1907-1930), par François SIMIAND (19321935), puis, de 1936 à 1957, par Émile COORNAERT. Une chaire Démographie sociale : la vie des populations lui a succédé et a eu pour titulaire Alfred SAUVY de 1959 à 1969. Cette chaire a été transformée en Sociologie de la civilisation moderne pour Raymond ARON de 1970 à 1978. Enfin a été créée, en 1981, une chaire Sociologie pour Pierre BOURDIEU (1981-2001). Une chaire Histoire moderne et contemporaine du politique a été créée en 2001 pour Pierre RosANVALLON. En 2005 est créée une chaire Rationalité et sciences sociales, occupée par Jon ELSTER (2006-2011) et en 2012 une chaire Sociologie du travail créateur, occupée par Pierre-Michel MENGER depuis 2013. En 2017 est créée une chaire Migrations et sociétés, confiée à François HÉRAN.

\section{Géographie}

Une chaire Géographie humaine, créée grâce à une libéralité d'Albert KAHN, a été occupée de 1912 à 1930 par Jean BRUNHES.

En 1932 était créée la chaire Géographie économique et politique, où enseigna André SIEGFRIED (1933-1946). La Géographie historique de la France fut enseignée, de 1892 à 1911, par Auguste LONGNON ; elle a été rétablie de 1947 à 1968 pour Roger DION, puis transformée en chaire Géographie du continent européen pour Maurice LE LANNOU, de 1969 à 1976. Une chaire Étude du monde tropical (géographie physique et humaine) a été créée en 1946 pour Pierre GouROU (1947-1970).

\section{Histoire des religions}

Une chaire Histoire des religions, créée en 1880, a eu pour titulaires Albert RÉVILLE (1880-1906), Jean RÉVILLE (1907-1908), Alfred LOISY (1909-1932), Jean BARUZI (1933-1951) et Henri-Charles PUECH (1952-1972).

Une chaire Histoire des mentalités religieuses dans l'Occident moderne a été créée en 1973 pour Jean DeluMEAU qui l'a occupée de 1975 à 1994 et une chaire Étude comparée des religions antiques en 1973 pour Jean-Pierre VERNANT, qui l'a occupée de 1975 à 1984.

En 1977, a été créée une chaire intitulée Christianisme et gnoses dans l'Orient préislamique pour Antoine GUILLAUMONT qui l'a occupée jusqu'en 1986 et en 1990 une chaire Histoire des syncrétismes de la fin de l'Antiquité pour Michel TARDIEU (1991-2008). En 2006 est créée une chaire intitulée Milieux bibliques pour Thomas 
RÖMER. Créée en 2014, une chaire Histoire du Coran. Texte et transmission est confiée en 2015 à François DÉROCHE.

\section{Préhistoire}

Fut créée en 1929 une chaire Préhistoire, tenue par l'abbé Henri BREUIL (1929-1947), discipline reprise de 1969 à 1982 par André LEROI-GOURHAN, puis transformée en 1982 en chaire Paléoanthropologie et préhistoire pour Yves COPPENS (1983-2005), elle-même transformée en chaire Paléontologie humaine en 2005 et occupée par Michel BRUNET (2007-2011). En 1993, une chaire Civilisations de l'Europe au néolithique et à l'âge du bronze a été créée pour Jean GUILAINE (19942007).

\section{Histoire générale}

L'histoire générale était professée au Collège de France depuis le dernier tiers du $\mathrm{XVIII}^{\mathrm{e}}$ siècle. On l'associait alors à la morale, et cette union persista, nominalement au moins, pendant tout le XIX ${ }^{\mathrm{e}}$ siècle. À Pierre DAUNOU (1819-1830), succédèrent Jean-Antoine LETRONNE (1831-1837), et Jules MiCHELET (1838-1852), puis, un peu plus tard, Joseph GUIGNIAUT (1857-1862), et Alfred MAURY (1862-1892).

Une chaire Histoire de la civilisation moderne tenue par Lucien FEBVRE (1933-1949), puis par Fernand BRAUDEL (1950-1972), subsiste pour Emmanuel LE ROY LADURIE, qui l'occupa de 1973 à 1999. En 1997, une chaire Histoire de la France des Lumières créée pour Daniel Roche (1999-2005), transformée en chaire intitulée Écrit et cultures dans l'Europe moderne pour Roger CHARTIER (2006).

Une chaire Civilisation indo-européenne a été occupée de 1948 à 1968 par Georges DuMÉZIL. Une chaire Histoire générale des sciences, créée en 1891, a été occupée par Pierre LAFFITTE (1892-1903), Grégoire WyRoubofF (1903-1913) et Pierre Boutroux (1920-1922). Une chaire Histoire globale de la première modernité est confiée en 2013-2014 à Sanjay SUBRAHMANYAM, avant d'être transformée en chaire pluriannuelle que Sanjay SUBRAHMANYAM occupe en tant que professeur invité (2014- ).

\section{Art}

Une chaire Esthétique et histoire de l'art, créée en 1878, a eu pour titulaires, successivement Charles BlanC (1878-1882), Eugène GuILlaume (1882-1905); Georges LAFENESTRE (1905-1919), André MiCHEL (1920-1925) (qui la spécialisa en Histoire de l'art français), Gabriel MiLlet (1926-1937), Henri FoCILLON (19381943). En 1970 a été créée une chaire Art et civilisation de la Renaissance en Italie pour André CHASTEL (1970-1984). Une des chaires fondées par la Ville de Paris, affectée en 1933 à Histoire de l'art monumental, a été occupée par Paul LÉON (19331944). Une autre a été affectée à Psychologie des arts plastiques pour René HUYGHE de 1951 à 1976 ; désormais chaire d'État, elle a été transformée en 1976 en Histoire de la création artistique en France pour Jacques THUILLIER, qui l'a occupée de 1977 à 1998. En 2000, une chaire Histoire de l'art européen médiéval et moderne a été créée pour Roland RECHT qui l'occupe de 2001 à 2012. 
Une chaire Invention, technique et langage en musique a été créée en 1975 pour Pierre BoulEz (1976-1995); elle fut transformée de 1996 à 1999 pour Jerzy GROTOWSKI en Anthropologie théâtrale.

\section{Mathématiques}

L'enseignement des mathématiques fut assuré par Antoine-Rémy MAUDUIT (1770-1815), Sylvestre-François LACROIX (1815-1843), Guillaume LIBRI-CARUCCI (1843-1848), Joseph LIOUVILLE (1851-1882), Camille JORDAN (1883-1912), Georges HuMBERT (1912-1921), Henri LEBESGUe (1921-1941). S'y ajoutait l'astronomie, professée par Jérôme DE LALANDE (1768-1807), puis par Jean-Baptiste DELAMBRE (1807-1822) et Jacques BINET (1823-1856). La chaire Astronomie fut transformée, en 1856, en Mécanique céleste pour Joseph SERRET (1861-1885). Elle devint chaire Mécanique analytique et mécanique céleste pour Maurice LÉVY (1885-1908), et Jacques HADAMARD (1909-1937). Une chaire Mathématique et mécanique a été occupée de 1938 à 1972 par Szolem MANDELBROJT, puis transformée en Analyse mathématique des systèmes et de leur contrôle pour Jacques-Louis LiONS (19731998). Une chaire Théorie des équations différentielles et fonctionnelles a été occupée par Jean LERAY de 1947 à 1978. Une chaire Algèbre et géométrie occupée par Jean-Pierre SERRE de 1956 à 1994 a été transformée, en 1995, en chaire intitulée Équations différentielles et systèmes dynamiques pour Jean-Christophe YoCCOZ (1996-2006). Une chaire Théorie des groupes a été créée pour Jacques TiTs (19732000) ainsi qu'une chaire Analyse et géométrie depuis 1983 pour Alain CONNES. En 1999, une chaire Théorie des nombres a été créée pour Don ZAGIER qui l'occupe de 2000 à 2014. Une chaire Équations aux dérivées partielles et applications a été créée en 2001 pour Pierre-Louis LIONS, qui l'occupe depuis 2002, et une chaire Géométrie algébrique en 2015 pour Claire VoISIN, qui l'occupe depuis 2016.

\section{Sciences numériques}

En 2011 est créée une chaire intitulée Algorithmes, machines et langages, confiée en 2012 à Gérard BERRY, en 2016 une chaire Sciences des données, occupée par Stéphane MALLAT depuis 2017, et en 2017 une chaire Sciences du logiciel, confiée à Xavier LEROY en 2018.

\section{Physique}

En 1769, une chaire Physique mathématique remplaça l'ancienne chaire Philosophie grecque et latine ; Jacques-Antoine Cousin l'occupa jusqu'en 1800 ; il eut pour successeur Jean-Baptiste BIOT, à la fois physicien et mathématicien (1801-1862).

Devenue chaire Physique générale et mathématique, elle fut occupée par Joseph BERTRAND (1862-1900), Marcel BRILlouin (1900-1931); sous le titre de Physique théorique, elle a été occupée par Léon BRILLOUIN (1932-1949) et par Jean LAVAL (1950-1970), puis transformée en 1971 en Physique de la matière condensée pour Pierre-Gilles DE GENNES (1971-2004). Cette chaire est occupée par un nouveau titulaire en 2009, Antoine GEORGES. D'autre part, en 1786, fut créée une chaire Physique générale et expérimentale, occupée par Louis LEFÈVRE-GINEAU jusqu'en 1823, André-Marie AMPÈRE (1824-1836), Félix SAVART (1836-1841), Henri-Victor RÉGNAULT (1841-1871), Élie MASCART (1872-1908), Paul LANGEVIN (1909-1946). À 
la chaire Physique générale et expérimentale, tenue par Maurice DE BROGLIE de 1942 à 1944 et sur laquelle fut réintégré Paul LANGEVIN, révoqué en 1940, succéda une chaire Physique atomique et moléculaire par transformation de la chaire Physique mathématique créée en 1933 pour Albert EINSTEIN et qu'il n'avait jamais occupée. Elle eut pour titulaire de 1946 à 1972 Francis PERRIN auquel succéda Claude COHENTANNOUDJ, de 1973 à 2004. Une nouvelle chaire Physique mathématique a été créée en 1951 pour André LICHNEROWICZ (1952-1986). Une chaire Chimie nucléaire occupée par Frédéric JOLIOT (1937-1958) a été transformée de 1958 à 1972 en Physique nucléaire pour Louis LEPRINCE-RINGUET puis en Physique corpusculaire de 1973 à 2004 pour Marcel FroISSART. Une chaire Physique cosmique, occupée de 1944 à 1962 par Alexandre DAUVILLIER, a été transformée en 1962 en Astrophysique théorique pour Jean-Claude PECKER (1964-1988), puis, en 1989, en Astrophysique observationnelle pour Antoine LABEYRIE (1991-2014). Une chaire Magnétisme nucléaire, créée en 1959, a été occupée par Anatole ABRAGAM jusqu'en 1985 et une chaire Méthodes physiques de l'astronomie par André LALLEMAND de 1961 à 1974. En 1982, une chaire Physique statistique a été créée pour Philippe NOZIÈRES (19832001). Une autre chaire Physique statistique est créée en 2014 pour Bernard DERRIDA.

Une chaire nouvelle, créée par la loi de Finances de 1964, et portant le titre de Physique théorique des particules élémentaires a été occupée par Jacques PRENTKI jusqu'en 1983. En 2000, une chaire Physique quantique a été créée pour Serge HAROCHE qui l'occupe depuis 2001. Gabriele VENEZIANO occupe de 2004 à 2013 la chaire Particules élémentaires, gravitation et cosmologie, créée en 2003. En 2005 est créée une chaire Physique mésoscopique, occupée par Michel DEVORET (20072012). En 2012, une chaire Atomes et rayonnement est confiée à Jean DALIBARD. En 2013, une chaire Galaxies et cosmologie est créée pour Françoise COMBES (2014), et en 2016 une chaire Champs, cordes et gravité pour Jean-Marc HENNEAUX (2017).

En 2017 est créée une chaire Matière molle et biophysique, confiée en 2018 à Jean-François JOANNY.

\section{Chimie}

Une chaire Chimie et une chaire Histoire naturelle, fondées en 1774, furent quelques temps réunies sous le titre de Chimie et histoire naturelle pour Jean DARCET, qui d'ailleurs limita son enseignement à la première de ces deux sciences. Le titre primitif de Chimie fut repris quand la chaire fut attribuée à Nicolas VAUQUELIN (1801-1804) auquel succéda Louis-Jacques THÉNARD (1804-1845). En 1845, elle se spécialisa sous le titre Chimie minérale, et elle eut pour titulaires Théophile-Jules PELOUZE (1845-1850), Antoine-Jérôme BALARD (1851-1876), Paul SCHÜTZENBERGER (1876-1897), Henri LE CHATELIER (1898-1907) et Camille MATIGNON (1908-1934). Une autre chaire, attribuée à la Chimie organique, eut pour premier titulaire Marcelin BERTHELOT (1865-1907), auquel succédèrent ÉmileClément Jungfleisch (1908-1916), Charles Moureu (1917-1929), Marcel DELÉPINE (1930-1941) et Charles DufRAISSE (1942-1955); elle a été transformée en 1955 en Chimie organique des hormones, pour Alain HoREAU (1956-1980). En 1979 a été créée pour Jean-Marie LEHN une chaire Chimie des interactions moléculaires (1979-2010), et de 1996 à 1998 pour Jean RoUXEL une chaire Chimie des solides. En 2000, une chaire Chimie de la matière condensée a été créée pour Jacques LiVAGE qui l'a occupé jusqu'en 2009. En 2007 est créée une chaire Chimie des processus biologiques pour Marc FONTECAVE. En 2011, une chaire Chimie des 
matériaux hybrides a été créée pour Clément SANCHEZ. Une chaire Chimie du solide et énergie, créée en 2012, est occupée par Jean-Marie TARASCON depuis 2013.

\section{Histoire naturelle et sciences biologiques}

L'enseignement de l'histoire naturelle fondé en 1774 n'étant pas effectivement donné par Jean DARCET, une chaire consacrée à la seule Histoire naturelle fut créée en 1778 et attribuée à Louis DAUBENTON (1778-1799), puis à Georges CUVIER (18001832). Cette chaire nouvelle ne tarda pas à être elle-même dédoublée. D'une part, une chaire Histoire naturelle des corps inorganiques fut donnée à Léonce ÉLIE DE BEAUMONT (1832-1874) et fut ensuite occupée par Charles SAINTE-ClaIRE DEVILLE (1875-1876), Ferdinand FouQUÉ (1877-1904), Auguste MICHEL-LÉVY (1905-1911). À cette chaire succèdent une chaire Géologie, occupée par Lucien CAYEUX (19121936) puis une chaire Géologie méditerranéenne pour Paul FALLOT (1938-1960) et une chaire Géodynamique pour Xavier LE PICHON (1986-2008). En 2000 est créée une chaire Évolution du climat et de l'océan pour Édouard BARD, et en 2011, une chaire Physique de l'intérieur de la Terre pour Barbara RoMANOwICZ. Entre-temps, une création temporaire de chaire consacrée aux Études coloniales, protistologie pathologique a eu pour titulaire Louis NATTAN-LARRIER (1923-1943). D'autre part, une seconde chaire fut consacrée à l'Histoire naturelle des corps organisés : elle eut pour titulaires Georges DUVERNOY (1837-1855), Pierre-Jean-Marie FlouRENS (18551867), Étienne-Jules MAREY (1869-1904), Nicolas FRANÇOIS-FRANCK (1905-1921) et André MAYER (1922-1946) ; elle a été transformée pour Jean ROCHE, de 1947 à 1972, en Biochimie générale et comparée, puis en Biochimie cellulaire pour François GROS (1973-1996) et enfin en 1996 en Immunologie moléculaire pour Philippe KOURILSKY (1998-2012). En 1980, une chaire Bioénergétique cellulaire a été créée pour Pierre JOLIOT (1981-2002). En 1995 a été créée pour Armand DE RICQLÈS (1995-2010) une chaire Biologie historique et évolutionnisme (1995-2010).

En 1844 fut créée une chaire Embryogénie comparée, tenue par Victor COSTE (1844-1873), Édouard BALBIANI (1874-1899), Félix HENNEGUY (1900-1928), puis par Emmanuel FAURÉ-FRÉMIET (1928-1954). Consacrée ensuite à l'Embryologie expérimentale, elle a été occupée de 1955 à 1974 par Étienne WoLfF. Depuis 1974 elle a été transformée en Communications cellulaires pour Jean-Pierre CHANGEUX (1976-2006).

Enfin, en 1964, une chaire nouvelle a été créée par la loi de Finances sous le titre de Génétique cellulaire pour François ЈАСОВ (1965-1991). Elle a été transformée en chaire Génétique moléculaire pour Pierre CHAMBON (1992-2002), puis en Génétique humaine pour Jean-Louis MANDEL (2003). En 1967 était créée pour Jacques MONOD une chaire Biologie moléculaire, qui l'occupa jusqu'en 1973. Une chaire Génétique et physiologie cellulaire a été créée en 2000 pour Christine PETIT, qui l'occupe depuis 2001, et une chaire Processus morphogénétiques est créée en 2006, occupée à partir de 2007 par Alain PROCHIANTZ. En 2012, une chaire Épigénétique et mémoire cellulaire est confiée à Edith HEARD et, créée en 2016, une chaire Dynamique du vivant, confiée à Thomas LECUIT en 2017.

\section{Médecine}

La médecine, enseignée au Collège dès le $\mathrm{XVI}^{\mathrm{e}}$ siècle, disposait de quatre chaires, progressivement spécialisées. 
L'anatomie fut professée par Antoine PORTAL de 1773 à 1832, tandis que la médecine dite pratique était attribuée à d'autres titulaires, parmi lesquels JeanNicolas CORVISART (1796-1804), Jean-Noël HALLÉ (1805-1822), René-Théophile LAENNEC (1822-1826), et Joseph RÉCAMIER (1827-1830). François MAGENDIE (1830-1855) eut pour successeurs Claude BERNARD (1855-1878), Charles BROWNSÉQUARD (1878-1894), Arsène D'ARSONVAL (1894-1930) et Charles NiCOLlE (1932-1936). Une chaire Épidémiologie fut créée pour Hyacinthe VINCENT (1925-1936). La chaire Médecine a été occupée par René LERICHE (1937-1950); transformée ensuite en Médecine expérimentale, elle a été occupée par Antoine LACASSAGNe (1951-1954), Charles OBERLING (1955-1960), Bernard HALPERN (1961-1975), Jean DAUSSET (1977-1987), et subsiste sous cette même dénomination pour Pierre CORVOL (1989-2012) et Alain FISCHER à partir de 2013. En 2014 est créée une chaire Oncologie cellulaire et moléculaire, confiée à Hugues DE THÉ.

Furent encore créées successivement plusieurs chaires nouvelles: en 1875, une chaire Anatomie générale, occupée par Louis-Antoine RANVIER (1875-1911), et transformée d'abord en Histologie comparée pour Jean NAGEOTTE (1912-1937) puis en Morphologie expérimentale et endocrinologie pour Robert COURRIER, de 1938 à 1966, puis en Physiologie cellulaire de 1967 à 1993 pour François MOREL ; enfin en chaire de Biologie moléculaire des plantes pour Joseph SCHELL de 1994 à 1998. En 1903, une chaire Pathologie générale et comparée fut créée pour Albert CHARRIN (1903-1907). Cette chaire fut transformée en Biologie générale, successivement occupée par le physiologiste Émile GLEY de 1908 à 1930 et par le physico-chimiste Jacques DUCLAUX de 1931 à 1948. Elle fut remplacée par une chaire Neurophysiologie générale pour Alfred FESSARD, de 1949 à 1971, et modifiée en 1971 en chaire Neurophysiologie pour Yves LAPORTE, de 1972 à 1991. Elle est devenue Physiologie de la perception et de l'action pour Alain BERTHOZ (1992-2010). En 1925, une chaire Histophysiologie, attribuée à Justin JOLLY (1925-1940), a été remplacée par une chaire Radiobiologie expérimentale, occupée par Antoine LACASSAGNE de 1941 à 1951 où il devint titulaire de la chaire Médecine expérimentale mentionnée au paragraphe précédent ; cette chaire a repris de 1951 à 1966, son ancienne affectation pour Jacques BENOIT ; elle est devenue en 1973 chaire Physiologie du développement pour Alfred JOST (1974-1987), puis, Embryologie cellulaire et moléculaire pour Nicole LE DOUARIN (1988-2000). En 1993, une chaire Fondements et principes de la reproduction humaine a été créée pour Étienne-Émile BAULIEU (1993-1998), transformée en 1998, en Biologie et génétique du développement pour Spyros ARTAVANIS-TSAKONAS qui l'occupe de 2000 à 2012. En 2006 est créée la chaire Microbiologie et maladies infectieuses pour Philippe SANSONETTI.

En 1929, une chaire Mécanique animale appliquée à l'aviation, attribuée à Antoine MAGNAN (1929-1938), a subsisté de 1939 à 1955 sous le titre d'Aérolocomotion mécanique et biologique, et a été occupée par Étienne OEHMICHEN.

À ces disciplines s'ajoutèrent en 1887 l'enseignement, alors nouveau, Psychologie expérimentale et comparée, confié successivement à Théodule RIBOT (1888-1901) et à Pierre JANET (1902-1934), puis les chaires Physiologie des sensations, attribuée à Henri PIÉRON (1923-1951), Psychologie et éducation de l'enfance occupée par Henri WALLON (1937-1949) ainsi que Neuropsychologie du développement créée en 1975 pour Julian DE AJURIAGUERRA qui l'occupa jusqu'en 1981 et qui a été transformée, en 1982, en chaire Neuropharmacologie pour Jacques GLOWINSKI (1983-2006). En 2005 est créée la chaire Psychologie cognitive expérimentale, confiée à Stanislas DEHAENE la même année. 


\section{CHAIRES INTERNATIONALES PLURIANNUELLES}

À l'assemblée des professeurs du 30 juin 2013 est décidée une nouvelle formule d'invitation de professeurs sur chaires pluriannuelles. La première chaire pluriannuelle est confiée, en 2014, pour trois ans, à Jean-Louis COHEN pour un cycle de cours intitulé : «Architecture et forme urbaine ». Sont ensuite nommés professeurs invités en 2014 Jean-Jacques Hublin (Paléoanthropologie) et Sanjay SubRAHMANYAM (Histoire globale de la première modernité, 2014-2019). L'assemblée du Collège de France, le 20 mars 2016, approuve le principe de création de chaires internationales accueillant des personnalités exerçant dans des institutions étrangères pour une durée de cinq ans. Elle crée, le 26 juin 2016, une chaire internationale intitulée «Histoire culturelle des patrimoines artistiques en Europe, $\mathrm{XVIII}$ - $\mathrm{XX}^{\mathrm{e}}$ siècle », nomme Bénédicte SAVOY sur cette chaire (2016-2021) et approuve la transformation des invitations sur chaires pluriannuelles de Jean-Louis COHEN et Jean-Jacques HuBLIN en chaires internationales d'une durée de cinq ans. En 2017, Denis Duboule est nommé enseignant invité sur une nouvelle chaire internationale intitulée «Évolution des génomes et développement» (2017-2022) et Edhem ELDEM sur une chaire internationale « Histoire turque et ottomane » (2017-2022).

\section{CHAIRES ANNUELLES}

\section{Chaire européenne (1989-2008)}

Cette chaire destinée à une personnalité scientifique originaire d'un pays membre de la communauté économique européenne, pour une année académique, a été créée en 1989. Elle a été occupée par Harald WEINRICH, professeur à l'université de Munich qui a traité de «Mémoire linguistique de l'Europe » pendant l'année 1989-1990. Le professeur Cesare VASOLI, de l'université de Florence, nommé pour l'année 19901991, n'a pu assurer son enseignement à la suite d'un accident de santé. En 1991-1992, Wolf LEPENIES, professeur au Wissenschaftskolleg de l'université libre de Berlin a traité du sujet suivant: "Les intellectuels et la politique de l'esprit dans l'histoire européenne »; en 1992-1993, Umberto ECO, professeur à l'université de Bologne, de «La quête d'une langue parfaite dans l'histoire de la culture européenne »; en 19931994, Werner HILDENBRAND, professeur à l'université de Bonn, du «Contenu empirique des théories économiques »; Norbert OHLER, professeur à l'université de Fribourg, en 1994-1995, de l'« Apport des pèlerins à la formation de l'Europe »; Klaus RAJEWSKI, professeur à l'université de Cologne, en 1995-1996, des « Nouvelles approches génétiques chez la souris »; Pieter WESTBROEK, professeur à l'université de Leyde, en 1996-1997, de "Géophysique: esquisse d'une nouvelle science de la Terre »; Abram DE SWAAN, professeur à l'université d'Amsterdam, en 1997-1998, de «Langue et culture dans la société transnationale»; Thomas W. GAEHTGENS, professeur à l'université libre de Berlin, en 1998-1999, de l' ' Image des collections en Europe au XVIII ${ }^{\mathrm{e}}$ siècle »; Hans-Wilhelm MÜLLER-GÄRTNER, professeur à la Faculté de médecine de Düsseldorf, en 1999-2000, des «Bases neuronales de la conscience : apport de l'imagerie cérébrale»; Michael EDWARDS, professeur à l'université de Warwick, en 2000-2001, de " Poétiques de l'anglais et du français »; Claudio MAGRIS, professeur à l'université de Trieste, en 2001-2002, de "Nihilisme et mélancolie. Jacobsen et son Niels Lyhne »; Hans BELTING, professeur à l'université de Heidelberg, en 2002-2003, de «L'histoire du regard. Représentation et vision en Occident»; 
Theodor BERCHEM, professeur émérite de l'université de Wurzbourg, de «L'avenir de l'université - l'université de l'Avenir », en 2003-2004 ; Sandro STRINGARI, directeur du Research and Development Center on Bose-Einstein Condensation à Trente, de «Condensation de Bose-Einstein et superfluidité », en 2004-2005. Les titulaires de cette chaire ont été ensuite, en 2005-2006, Maurice BLOCH: «L'anthropologie cognitive à l'épreuve du terrain »; en 2006-2007, Guy ORBAN : «La vision, mission du cerveau »; en 2007-2008, Manfred KROPP : «Études coraniques ».

\section{Chaire Développement durable - Environnement, énergie et société (2008-2016)}

En 2008-2009, la chaire européenne est thématisée «Développement durable - Environnement, énergie et société » et reçoit le soutien de TOTAL. Elle est confiée à Henri LERIDON : "Démographie, fin de la transition », puis est occupée par Nicolas STERN en 2009-2010 : «Gérer les changements climatiques, promouvoir la croissance, le développement et l'équité »; par Jean-Marie TARASCON, en 20102011 : « Enjeux et défis du stockage et de la conversion de l'énergie dans le contexte du développement durable »; par Paul ColONNA en 2011-2012: «Enjeux et défis pour le développement durable», par Annie CAZENAVE en 2012-2013 : «Étude de la terre et de l'environnement depuis l'espace »; par Gilles BoEUF en 2013-2014 : « La biodiversité, son évolution et ses croisements avec l'humanité »; par Georges CALAS en 2014-2015 : «Les ressources minérales, un enjeu majeur dans le contexte du développement durable »; par Thomas STERNER, enfin, en 2015-2016 : «Pour une politique économique de l'environnement : une approche conceptuelle ».

\section{Chaire internationale (1992-2008)}

Créée en 1992 pour accueillir, pendant une année académique, des personnalités scientifiques originaires des pays de l'Europe de l'Est ou appartenant à d'autres continents, cette chaire a eu pour premier titulaire Bronislaw GEREMEK, professeur à l'Académie des sciences de Varsovie, qui a traité en 1992-1993 du sujet suivant: «Histoire sociale: exclusions et solidarités ». En 1993-1994, ZHANG Guangda, professeur à l'université de Pékin, a traité de «La Chine et les civilisations de l'Asie centrale du VII ${ }^{\mathrm{e}}$ au XI ${ }^{\mathrm{e}}$ siècle »; Orest RANUM, professeur à l'université Johns Hopkins de Baltimore, en 1994-1995, de «La France des années 1650; histoire et historiographie »; Harris MEMEL-FOTÊ, professeur à l'université d'Abidjan, en 19951996, de «L'esclavage lignager africain et l'anthropologie des droits de l'Homme »; Igor MEL'ČUK, professeur à l'université de Montréal, en 1996-1997, de «Linguistique "Sens-Texte" »; Brian STOCK, professeur à l'université de Toronto, en 1997-1998, de «La connaissance de soi et la littérature autobiographique au Moyen Âge »; Patrice HigonNET, professeur à l'université d'Harvard, en 1998-1999, de « Mythes de Paris, des Lumières au surréalismes »; James Watson CRONIN, professeur à l'université de Chicago, en 1999-2000, de «Développement de la physique des particules et des grandes expériences »; Miklós SZABÓ, professeur à l'université Eötvös Loránd de Budapest, en 2000-2001, de l' « Histoire des Celtes danubiens et leur romanisation »; Paul FARMER, professeur à la Harvard Medical School de Boston, en 2001-2002, de «La violence structurelle et la matérialité du social »; Stuart EDELSTEIN, professeur à l'université de Genève, en 2002-2003, de « Mécanismes de la transduction du signal en biologie ». Jayant Vishnu NARLIKAR, professeur à l'Inter-University centre for 
Astronomy and Astrophysics de Pune (Inde) a traité en 2003-2004 de « Cosmology: Theory and observations »; A.M. Celâl ŞENGÖR, professeur à l'université technique d'Istanbul, en 2004-2005, de «L'histoire de la tectonique depuis les temps les plus reculés jusqu'à l'apparition de la tectonique des plaques : une étude épistémologique ». Les titulaires de cette chaire ont été ensuite, en 2005-2006, Thomas PAVEL: «Comment écouter la littérature ? »; en 2006-2007, Daniele VITALI : « Les Celtes d'Italie »; en 2007-2008, Pierre MAGISTRETTI : «Cellules gliales, neuroénergétique et maladies neuropsychiatriques ».

\section{Chaire Savoirs contre pauvreté (2008-2014)}

En 2008-2009, la chaire internationale est thématisée « Savoirs contre pauvreté » et reçoit le soutien de l'AFD (Agence française de développement) jusqu'en 2012. Elle est tout d'abord confiée à Esther DUFLO : «Pauvreté et développement dans le monde », puis est occupée par Peter PIOT en 2009-2010 : «L'épidémie du Sida et la mondialisation des risques »; par Ismail SERAGELDIN, en 2010-2011 : « La faim et la sécurité alimentaire dans le monde »; par Manuela CARNEIRO DA CUNHA en 2011-2012 : « Savoirs autochtones : nature et apports »; par Dominique KEROUEDAN en 2012-2013 : « Géopolitique de la santé mondiale »; par François BouRGUIGNON en 2013-2014 : «Pauvreté, développement et mondialisation », dernier titulaire de cette chaire.

\section{Chaire de création artistique (2004- )}

Créée en 2004 pour accueillir, pendant une année académique, une personnalité illustrant la création artistique contemporaine, et consacrée à toutes les formes de création artistique, cette chaire a eu pour premier titulaire, en 2005-2006, Christian DE PORTZAMPARC, architecte, qui a traité de : «Architecture : figures du monde, figures du temps». La chaire est ensuite occupée, en 2006-2007, par Pascal DUSAPIN, compositeur, dont le cours a pour titre : "Composer : musique, paradoxe, flux ». En 2007-2008, la chaire est confiée à Ariane MNOUCHKINE (non occupée) ; en 2008-2009 à Pierre-Laurent AIMARD: "Paramètres et dimensions de l'interprétation musicale »; en 2009-2010 à Jacques NICHET : «Le théâtre n'existe pas »; en 2010-2011 à Anselm KIEFER : «L'art survivra à ses ruines »; en 20112012 à Gilles CLÉMENT : « Jardin, paysage et génie naturel »; en 2012-2013 à Karol BEFFA : «Musique : art, techniques, savoirs »; en 2013-2014 à Tony CRAGG : « Sculpture et langage »; en 2015-2016, à Alain MABANCKOU : «Lettres noires : des ténèbres à la lumière »; en 2016-2017, à Philippe MANOURY : "L'invention de la musique ».

\section{Chaire d'innovation technologique Liliane Bettencourt (2007- )}

Créée en 2007, en partenariat avec la fondation Bettencourt-Schueller, cette chaire a pour vocation d'accueillir, pour chaque année académique, un nouveau titulaire chargé de proposer un enseignement à la pointe de la recherche dans les secteurs hautement innovants des nanotechnologies, de l'informatique, des réseaux de communication, du transfert et du cryptage de données, des sciences du vivant. Le premier titulaire, en 2006-2007, a été Jean-Paul CLOZEL, chercheur et P.-D.G. de la société Actelion Ltd, qui a traité de: «La biotechnologie: de la science au médicament ». En 2007-2008, la chaire est occupée par Gérard BERRY, directeur 
scientifique d'Esterel Technologies, qui s'est consacré à la question : «Pourquoi et comment le monde devient numérique ». Lui succèdent, pour 2008-2009, Mathias FINK, professeur à l'École supérieure de physique et de chimie industrielles de la ville de Paris et directeur du laboratoire Ondes et acoustique : «Ondes et images », et pour 2009-2010, Patrick COUVREUR, professeur des universités : "Les nanomédicaments ». En 2010-2011, Elias ZERHOUNI, professeur à la Johns Hopkins University (États-Unis), occupe la chaire et son cours porte sur «Les grandes tendances de l'innovation biomédicale au XXI ${ }^{\mathrm{e}}$ siècle ». En 2011-2012, Jean-Paul LAUMOND, directeur de recherche au CNRS lui succède avec un cours intitulé «Robotique : les fondations d'une discipline». En 2012-2013, Yves BRÉCHET, professeur à Grenoble-INP propose un cours portant sur: «La science des matériaux : du matériau de rencontre au matériau sur mesure». En 2013-2014, Philippe WALTER, directeur du laboratoire d'archéologie moléculaire et structurale à l'université Pierre et Marie Curie, lui succède avec un cours sur « Chimie analytique et histoire de l'art », puis, en 2014-2015, Bernard MEUNIER, directeur de recherche émérite au CRNS, avec un cours sur "Innovations thérapeutiques : évolutions et tendances »; en 2015-2016, José-Alain SAHEL : «Voir encore. La restauration visuelle en perspectives »; en 2016-2017, Didier RouX : « Recherche fondamentale, inventions et innovations »; en 2017-2018, Thomas EBBESEN : «L'Alchimie du vide : interactions lumière-matière en chimie physique ».

\section{Chaire Informatique et sciences numériques (2009- )}

Créée en 2009, en partenariat avec l'Institut national de recherche en informatique et en automatique (Inria), cette chaire accueille chaque année un nouveau titulaire. Le premier titulaire en 2009-2010, a été Gérard BERRY, directeur scientifique d'Esterel Technologies, dont le cours a porté sur : «Penser, modéliser et maîtriser le calcul informatique ». En 2010-2011, Martin ABADI, professeur à l'université de Californie (Santa Cruz), occupe la chaire et son cours porte sur «La sécurité informatique ». Lui succède en 2011-2012 Serge ABITEBOUL, directeur de recherche en informatique et en automatique, avec un cours intitulé «Sciences de données : de la logique du premier ordre à la Toile ». Bernard CHAZELLE, professeur à l'université de Princeton, l'occupe en 2012-2013 pour un cours intitulé « L'algorithmique et les sciences ». Nicolas AYACHE, directeur de recherche à Inria, lui succède en 20132014 avec un cours intitulé : « Le patient numérique personnalisé : images, médecine et informatique », Marie-Paule CANI, avec un cours sur « Façonner l'imaginaire : de la création numérique 3D aux mondes virtuels animés » (2014-2015), puis Yann LECUN : «L'apprentissage profond: une révolution en intelligence artificielle » (2015-2016) ; Jean-Daniel BoISSONNAT : «Géométrie algorithmique : des données géométriques à la géométrie des données »; Claire MATHIEU : «Algorithmes » (2017-2018).

\section{Chaire européenne (2016- )}

Créée en 2016, cette chaire consacrée à l'Europe a accueilli Alain WIJFFELS en 2016-2017, pour un cours intitulé «Miroir et mémoire de l'Europe : à la recherche d'une culture juridique partagé, du Moyen Âge à l'époque contemporaire », et Victor StoichitA, en 2017-2018, pour un cours sur «L'Europe des images ». 


\section{Missions et organisation du Collège de France}

De l'historique des chaires, il ressort que le Collège de France a souvent introduit des enseignements qui n'avaient pas encore reçu ailleurs droit de cité. C'est ce qui a fait dire à Ernest RENAN qu' «à côté des établissements où se garde le dépôt des connaissances acquises, il est donc nécessaire qu'il y ait des chaires indépendantes où s'enseignent, non les branches de la Science qui sont faites, mais celles qui sont en voie de se faire ».

Le Collège de France recrute sans condition de grades universitaires ; et par là, il lui est possible d'appeler à lui des savants qui ne sont pas des professeurs de carrière mais qui se sont signalés par des découvertes, par des vues personnelles, par des travaux originaux. Il suffit qu'on soit en droit d'attendre d'eux, dans le domaine de leurs recherches propres, des résultats nouveaux.

En outre, il ne prépare à aucun examen et, par conséquent, ses enseignements ne sont assujettis à d'autre programme que celui défini chaque année par le titulaire de la chaire et approuvé par l'assemblée du Collège de France. Nulle part, la recherche scientifique ne jouit d'une indépendance aussi large. De plus en plus, cette liberté est devenue sa loi, parce qu'elle est sa raison d'être ; et, de plus en plus, elle a déterminé son organisation. Les statuts de l'établissement sont régis par le décret no $2014-838$ du 24 juillet 2014 relatif au Collège de France (décret reproduit dans l'Annuaire du Collège de France 2014-2015, Paris, Collège de France, 2016, p. 73-81).

N'étant pas contraint à des cycles d'études invariables, le Collège de France n'a pas, en principe, de thématiques permanentes. Les enseignements anciens peuvent disparaître ou se transformer, des enseignements nouveaux peuvent être institués. Le nombre statutaire de possibilités de chaires de professeurs titulaires est actuellement de cinquante-sept. Les chaires du Collège de France sont créées par le ministre chargé de l'Enseignement supérieur sur proposition de l'assemblée du Collège de France. Cette proposition comporte le nouveau projet d'enseignement et de recherche ainsi que l'intitulé de la chaire. La création de la chaire s'accompagne de la publication au Journal officiel de la République française d'un avis de vacance. Les candidats adressent à l'administrateur du Collège de France leur déclaration de candidature et l'exposé de leurs titres et travaux. Après avoir examiné les candidatures et recueilli l'avis de l'Académie de l'Institut de France compétente, l'Assemblée propose un candidat au ministre chargé de l'Enseignement supérieur. Le professeur titulaire d'une chaire du Collège de France est nommé par décret du président de la République, sur le rapport du ministre chargé de l'Enseignement supérieur.

À partir de 1970, le principe d'invitation de conférenciers a été admis. Des savants étrangers sont ainsi invités chaque année par l'Assemblée à venir au Collège donner, pendant un ou deux mois, un enseignement relatif à leurs recherches.

Par ailleurs, deux chaires, créées en 1989, ont permis l'accueil de savants étrangers pour la durée d'une année académique :

- une chaire dite «européenne»(1989-2008), destinée à une personnalité scientifique originaire d'un pays membre de la Communauté économique européenne ; cette chaire devient, en 2008, chaire «Développement durable Environnement, énergie et société » (2008-2016) ;

- en 1991, une chaire dite «internationale», destinée à une personnalité originaire des pays de l'Europe de l'Est ou d'autres continents (1989-2008), transformée en 2008 en chaire « Savoirs contre pauvreté » (2008-2014). 
En 2004 est créée une chaire de création artistique, consacrée à toutes les formes de création artistique, qui accueille différents artistes sur une année académique Et d'autres chaires annuelles sont mises en place les années suivantes : en 2007, la chaire d'Innovation technologique Liliane Bettencourt, en partenariat avec la fondation Bettencourt-Schueller, et en 2009 une chaire d'Informatique et sciences numériques, en collaboration avec l'Institut national de recherche en informatique et en automatique (Inria).

En 2013, l'assemblée du Collège de France adopte une nouvelle formule d'invitation de professeurs sur plusieurs années. Ce dispositif permet d'inviter au Collège de France pour un enseignement relativement long des professeurs qui ne souhaitent pas renoncer à leur poste permanent dans une autre institution. À ces nouvelles chaires «internationales» pluriannuelles (cinq ans) s'ajoute enfin, en 2016, une chaire annuelle consacrée à l'Europe, dite « chaire européenne ».

Dans l'enseignement, comme dans la création et le renouvellement des chaires, prédomine aussi le principe de liberté. Chaque professeur choisit, d'année en année, le sujet de son cours dans le domaine scientifique qui lui est propre, et généralement dans l'ordre particulier de recherches auxquelles il s'applique à ce moment. Il le soumet ensuite à l'approbation de l'assemblée du Collège. Une partie de l'enseignement peut être donnée dans des institutions françaises en dehors de Paris, en France ou dans d'autres pays ; cette possibilité a été étendue à l'ensemble des établissements d'enseignement supérieur européens à partir de 1989, extraeuropéens à partir de 1992.

Les cours du Collège étant ouverts à tous, il n'y a ni inscription ni droits à payer. L'accès des salles d'enseignement est entièrement libre, dans la limite des places disponibles.

C'est à l'assemblée du Collège de France qu'appartiennent, sous réserve du contrôle de conformité ministériel, toutes les décisions relatives aux intérêts généraux de l'établissement. L'exécution de ces décisions et la direction des services sont confiées à un administrateur. Ce dernier doit être élu parmi les professeurs en exercice. Il est présenté par ses collègues et nommé par trois ans par décret du président de la République, sur le rapport du ministre. Il préside l'Assemblée, dont le bureau comprend, à ses côtés, le vice-président et le secrétaire de l'Assemblée, choisis l'un et l'autre parmi les professeurs et nommés par arrêté de l'administrateur, dans les conditions fixées par l'Assemblée.

Par une loi du 31 décembre 1932, l'établissement, qui déjà était investi de la personnalité civile, a été également doté de l'autonomie financière. Le décret du 24 juillet 2014 relatif au Collège de France abroge les textes antérieurs (décrets de 1911 et de 1990). Il rationalise le corpus réglementaire applicable à l'institution. Il préserve les missions du Collège de France et affirme son statut d'établissement public à caractère scientifique, culturel et professionnel, sous la forme d'un grand établissement, et son organisation collégiale.

L'assemblée du Collège de France comprend les professeurs titulaires. Les professeurs honoraires, les professeurs sur chaire annuelle, les professeurs invités sur une base annuelle ou pluriannuelle peuvent être conviés par l'administrateur à assister à l'assemblée. Ils n'ont pas voix délibérative et ne peuvent intervenir que sur invitation de l'administrateur. Conformément à l'article L. 953-2 du Code de l'éducation, le directeur général des services et l'agent comptable participent avec 
voix consultative aux instances administratives de l'établissement. En outre, l'administrateur peut inviter des personnalités à assister aux séances de l'Assemblée, et à y intervenir sur sa demande, sans y avoir voix délibérative. L'assemblée du Collège de France détermine la politique de l'établissement. Elle exerce les compétences conférées au conseil d'administration des universités notamment par le IV de l'article L. 712-3 du Code de l'éducation ainsi que celles conférées au conseil académique et à ses commissions par l'article L. 712-6-1 du même code. L'avis du Conseil d'établissement - qui comprend, outre l'administrateur, neuf professeurs, quinze représentants élus des personnels et cinq personnalités extérieures - doit précéder la délibération de l'Assemblée dans les matières relevant de la compétence du conseil d'administration. Créé le 18 avril 2010 par un arrêté de l'administrateur après une délibération à l'unanimité de l'Assemblée du Collège de France du 21 mars 2010, le comité technique paritaire (CTP) - devenu comité technique (CT) par la loi du 5 juillet 2010 sur la rénovation du dialogue social - constitue le lieu privilégié du dialogue social au sein du Collège de France. Cet organe consultatif, composé de dix membres titulaires, est présidé par l'administrateur. Il se réunit au moins deux fois par an et ses avis sont transmis à l'Assemblée. Le comité d'hygiène, de sécurité et des conditions de travail, composé de dix membres titulaires, apporte son concours dans les matières relevant de sa compétence au comité technique de l'établissement, auquel il rend compte de son activité.

Le Comité international d'orientation scientifique et stratégique (COSS), créé par l'assemblée du Collège de France en 2003, est pris en compte dans le décret du 24 juillet 2014. Il a pour rôle d'analyser les orientations scientifiques et stratégiques du Collège de France et les conditions d'exercice de ces missions, afin de proposer des recommandations d'évolution ou de mise en valeur de celles-ci. Il examine également la cohérence de la stratégie de l'institution, la signification et l'exploitation de la multidisciplinarité des chaires, la politique internationale, l'adéquation des moyens de recherche aux objectifs fixés ainsi que l'optimisation des structures administratives. Ses membres, au nombre de 12, exercent leur activité dans des universités ou institutions étrangères et ont été renouvelés à deux reprises. Le COSS est composé, depuis 2017, des membres suivants : John BAINES, université d'Oxford (Royaume-Uni), Jens BECKERT, Max Planck Institute for the Study of Societies (Allemagne), Yves-Alain BoIs, Institute of Advanced Studies de Princeton (ÉtatsUnis), Georges BROOKE, université de Manchester (Royaume uni), Avelino CORMA, CSIC-Polytechnical University of Valencia (Espagne), Nicola MARGOT COURTRIGHT, Amherst College (États-Unis), Luiz DAVIDOvich, université fédérale de Rio de Janeiro (Brésil), Peter Goodrich, Yeshiva University, New York (États-Unis), William HANKS, université de Californie, Berkeley (États-Unis), Ann JEFFERSON, université d'Oxford (Royaume uni), Edward LEE, université de Californie, Berkeley (États-Unis), Hélène REY, London Business School (Royaume uni), Wolf SINGER, Ernst Strüngmann Institute, Frankfort-sur-le-Main (Allemagne). 


\section{Les ADMinistrateurs du COLLĖGE dE FRANCE ${ }^{3}$}

Louis LeFÈVRe-GineAU (1800-1823), Isaac SiLVeSTRE DE SACY (1824-1838), Louis THÉNARD (1838-1840), Jean-Antoine LETRONNE (1840-1848), Jules BARTHÉLÉMY SAINT-Hilaire (1848-1852), Xavier DEPORTETS (1852-1853), Wilhelm RINN (1853-1854), Stanislas JULIEN (1854-1873), Édouard LABOULAYE (1873-1883), Ernest RENAN (1883-1892), Gaston BoISSIER (1892-1894), Gaston PARIS (1894-1903), Émile LEVASSEUR (1903-1911), Maurice CROISET (1911-1929), Joseph BÉDIER (1929-1936), Edmond FARAL (1937-1954), Marcel BATAILlON (1955-1965), Étienne WOLFF (1966-1974), Alain HoREAU (1974-1980), Yves LAPORTE (1980-1991), André MIQUEL (1991-1997), Gilbert DAGRON (1997-2000), Jacques Glowinski (2000-2006), Pierre CoRvol (2006-2012), Serge HAROCHE (2012-2015), Alain PROCHIANTZ (2015-).

\section{FONDATIONS}

\section{Fondation Hugot du Collège de France}

En 1977, l'assemblée des professeurs du Collège de France décidait à l'unanimité de créer une fondation dite «fondation Hugot du Collège de France » consacrée, conformément aux intentions de ses bienfaiteurs, Hélène et Jean-Pierre Hugot, à favoriser au mieux de ses moyens et en étroit accord avec le Collège de France, « la rencontre de diverses disciplines qui œuvrent à la connaissance, à la formation et à l'épanouissement de l'homme, le rapprochement, par-delà toutes les frontières, des meilleurs esprits animés de ce même souci ». Pour ce faire, la Fondation s'efforce de développer toutes études, recherches et activités ayant un double caractère à la fois humaniste et pluridisciplinaire.

La fondation Hugot promeut la coopération scientifique internationale en hébergeant les savants étrangers invités par l'assemblée des professeurs du Collège de France et en accueillant des réunions de travail et des colloques restreints organisés par un professeur. Elle accorde également des subventions de recherche et finance de nombreux colloques et événements culturels organisés pour la plupart au Collège de France.

Le Conseil d'État, par décret en date du 7 février 1979, faisait droit à la demande de l'Assemblée reconnaissant comme établissement d'utilité publique autonome ladite Fondation.

En 2001, le Conseil d'administration de la Fondation a décidé de la création d'un «Prix de la fondation Hugot du Collège de France ». Ce «prix Hugot», attribué par l'assemblée des professeurs du Collège de France, financé par la fondation Hugot du Collège de France, récompense chaque année deux jeunes chercheurs, en général de disciplines différentes, sélectionnés parmi les maîtres de conférences associés et les ATER (attachés temporaires d'enseignement et de recherche) rattachés au Collège de France durant l'année précédente.

3. Liste des administrateurs depuis l'institution de ce titre. 


\section{Prix de la fondation Hugot du Collège de France}

Le premier lauréat, pour l'année 2003-2004, est Ricardo URIBE-VARGAS, chaire Équations différentielles et systèmes dynamiques. Les lauréats suivants sont: Han HEE-JIN, chaire Philosophie des sciences biologiques et médicales (2004-2005); Wouter HENKELMAN, chaire Histoire et civilisation du monde achéménide et de l'Empire d'Alexandre (2005-2006) ; Jacques BOUTET DE MONVEL, chaire Génétique et physiologie cellulaire (2007-2008) ; Igor DotSENKo, chaire Physique quantique (2008-2009); Luca D’AMBROSIO, chaire Études juridiques comparatives et internationalisation du droit, et Nicolas RoCH, chaire Physique mésoscopique (20092010) ; Émilie FRENKIEL, chaire Histoire moderne et contemporaine du politique, et Matthieu VERNET, chaire Littérature française moderne et contemporaine : histoire, critique, théorie (2010-2011) ; Béatrice LIETZ, chaire Religion, institutions et société de la Rome antique, et Jernej MrAVLJE, chaire Physique de la matière condensée (2011-2012) ; Abhishek BANERJEE, chaire Analyse et géométrie, et Yuji MURAKAMI, chaire Littérature française moderne et contemporaine (2012-2013); Lauriane Chomaz, chaire Atomes et rayonnement, et Caroline DEVAUX, chaire État social et mondialisation: analyse juridique des solidarités (2013-2014); Sandra ZANELLA, chaire Techniques et économies de la Méditerranée antique, et Jonathan ZAPATA, chaire Processus morphogénétiques (2014-2015); Jean-Baptiste GuILLON, chaire Métaphysique et philosophie de la connaissance, et Simon HENRY, chaire Analyse et géométrie (2015-2016) ; Adrien GENOUDET, chaire Histoire des pouvoirs en Europe occidentale, $\mathrm{XIII}^{\mathrm{e}}-\mathrm{XVI}^{\mathrm{e}}$ siècle, et Tania TODOROVA YorDANOVA, chaire Chimie des processus biologiques (2016-2017) ; Sanchit KUMAR, chaire Histoire intellectuelle de la Chine, et Sarah TESSIER, chaire Oncologie cellulaire et moléculaire.

\section{Fondation du Collège de France}

La vocation de la Fondation du Collège de France ${ }^{4}$, reconnue d'utilité publique, est de soutenir la recherche scientifique et d'accompagner le Collège de France dans sa mission de diffusion des connaissances auprès du plus grand nombre. Pour cela, la fondation fait appel à la générosité des particuliers et des entreprises.

Le mécénat et les dons collectés permettent de répondre aux besoins de financement de projets de recherche des professeurs, d'accroître les ressources nécessaires à l'animation de la vie scientifique des chaires, d'acquérir des équipements techniques de dernière génération, d'amplifier la formation des jeunes chercheurs, de favoriser l'accès aux connaissances et aux dernières recherches menées par les chaires et par les équipes accueillies.

La gouvernance de la Fondation du Collège de France est assurée par un conseil d'administration qui définit la politique générale de la fondation, fixe les orientations stratégiques, vote les budgets et approuve les comptes. Constitué de 12 membres, il associe des professeurs du Collège de France, des personnalités qualifiées ainsi que des représentants des donateurs et des mécènes. Cette composition illustre la volonté de dialogue et d'échange de la fondation avec la société civile et les acteurs du monde économique. Le Conseil d'administration est assisté d'un Comité

4. Pour plus d'informations : www.fondation-cdf.fr. 
d'orientation scientifique composé de 6 à 8 membres issus du corps professoral du Collège de France.

Le décret de création de la Fondation du Collège de France a été publié au Journal officiel du 7 avril 2008.

\section{Bourse Anna Caroppo - Fondation du Collège de France}

Créée en 2017 à la faveur du don de M. Lucio ToscANO, grand donateur de la Fondation du Collège de France, la bourse Anna Caroppo - Fondation du Collège de France accompagne de jeunes chercheuses en leur permettant de poursuivre, pendant une année, leur formation à la recherche en sciences humaines au sein des équipes du Collège de France.

Les lauréates sont : Hengying Rong (2017), Camila PERruso (2018), Emmanuelle PORTUGAL (2018).

\section{PARTENARIATS}

\section{Fondation de coopération scientifique « Paris Sciences et Lettres - Quartier latin »}

«Paris Sciences et Lettres - Quartier latin» (dit PSL) est une fondation de coopération scientifique au sens de l'article L. 344-1 du Code de la recherche, régie par les articles L. 344-11 à L. 344-16 du même code. Ses statuts initiaux ont été approuvés par un décret daté du 8 juillet 2010 ; ses statuts modifiés ont été approuvés par un décret du 3 septembre 2015. Cette fondation a pour objet d'assurer la coopération scientifique entre les établissements d'enseignement supérieur et de recherche qui en sont membres, fondateurs et associés.

Cette coopération a pour but la création d'une université de recherche fondée sur une vision partagée par les institutions membres, c'est-à-dire un pôle commun structurant une convergence de recherche, de valorisation et de formation par la recherche, dans le respect de l'autonomie de chaque membre, fondateur ou associé. Cette université de recherche vise à 1) développer, améliorer et renforcer les performances de recherche, d'éducation et de diffusion des savoirs grâce aux synergies entre établissements ; 2) promouvoir la formation par la recherche à tous les niveaux et pour tous les objectifs de formation, et susciter de la sorte un effet d'entraînement pour tout le système d'enseignement supérieur français ; 3) renforcer les interactions entre la recherche académique et les besoins socio-économiques, PSL agissant comme un moteur de la croissance économique et de l'innovation.

La fondation est la structure qui porte la création d'une université de recherche constituée par le pôle scientifique commun. À ce titre, elle exerce les compétences et met en œuvre les activités communes que lui confient ses membres. Elle assure la gestion des équipements ou des services mutualisés que ses membres lui confient, et peut être chargée de la coordination des projets immobiliers communs à ses membres.

Les membres fondateurs sont le Collège de France, l'École nationale supérieure de chimie de Paris (ENSCP, Chimie ParisTech), l'École nationale supérieure des mines de Paris (Mines ParisTech), l'École normale supérieure, l'École supérieure de physique et de chimie industrielles (ESPCI ParisTech), l'institut Curie, l'Observatoire 
de Paris, l'université Paris-Dauphine, l'association Art et Recherche, le Centre national de la recherche scientifique et l'Institut national de la santé et de la recherche médicale. Les membres associés sont : l'École française d'Extrême-Orient, l'École des hautes études en sciences sociales, l'École nationale des Chartes, l'École nationale supérieure des arts décoratifs, l'École nationale supérieure des beaux-arts, l'École nationale supérieure des métiers de l'image et du son (la Fémis), l'École pratique des hautes études, le Conservatoire national supérieur de musique et de danse de Paris, le Conservatoire national supérieur d'art dramatique, l'Institut de biologie physico-chimique - fondation Edmond de Rothschild, l'institut Louis Bachelier, la fondation Pierre-Gilles de Gennes pour la recherche, l'Institut national de recherche en informatique et en automatique ${ }^{5}$.

PSL a été lauréat, en 2010, de l'opération Campus dont le Collège de France a bénéficié pour réaliser la rénovation-restructuration du site Cardinal-Lemoine et en juillet 2011, de l'IDEX (Initiative d'excellence du plan d'investissement). En 2013, les membres de PSL ont signé l'accord de consortium qui les engage à mettre en œuvre la stratégie définie dans le projet d'IDEX.

Par décret ministériel en date du 28 juillet 2016, publié au Journal officiel du 30 juillet 2016, sont approuvées les modifications apportées aux statuts de la fondation de coopération scientifique (FCS) «Paris sciences et lettres - Quartier latin », telles qu'adoptées par son conseil d'administration, en sa séance du 24 mars 2016. L'Institut Pasteur est ajouté à la liste des membres associés (décret du Premier ministre en date du 2 septembre 2016, publié au Journal officiel du 4 septembre 2016).

Le décret d'association de la communauté d'universités et établissements (ComUE) Université de recherche Paris sciences et lettres - PSL Research University fixe les compétences mises en commun entre les établissements partenaires dans le cadre de la convention d'association qui les lient. Elles concernent une communication de site commune ainsi que la signature des publications scientifiques sous l'identité «PSL Research University », la recherche scientifique, la politique internationale, la formation doctorale, la qualité de vie étudiante et de promotion sociale ainsi que l'insertion professionnelle des étudiants.

Le texte final de l'accord d'association avec l'université PSL est approuvé par l'assemblée du Collège de France le 26 mars 2017.

\section{Programme Pause}

Le Programme national d'aide à l'Accueil en urgence des scientifiques en exil (Pause), créé le 16 janvier 2017, accorde des financements incitatifs aux établissements d'enseignement supérieur et aux organismes de recherche publics projetant d'accueillir des scientifiques en situation d'urgence et les accompagne dans leurs démarches.

Annoncé en octobre 2016 par le secrétaire d'État chargé de l'Enseignement supérieur et de la Recherche, à l'occasion du colloque «Migrations, réfugiés, exil » du Collège de France, le programme PAUSE est un programme national ambitieux,

5. Pour la liste des membres : Statuts de la fondation de coopération scientifique «Paris Sciences et Lettres - Quartier latin », version modificative du 16 décembre 2014 approuvée par décret du 3 septembre 2015. 
initié par l'État avec l'appui de la société civile et des acteurs économiques. Il a pour mission de favoriser l'accueil des scientifiques en situation d'urgence sur des périodes suffisamment longues pour permettre leur intégration et assurer la continuité de leurs travaux. PAUSE est soutenu par un comité de parrainage engagé et prestigieux, co-présidé par Alain Prochiantz, administrateur du Collège de France, et Edith Heard, professeure au Collège de France.

Lancé officiellement le 16 janvier 2017, à l'occasion de la signature d'une convention entre le ministère de l'Éducation nationale, de l'Enseignement supérieur et de la Recherche, le Collège de France et la Chancellerie des universités de Paris, le programme national Pause réunit les grandes institutions de la communauté de l'enseignement supérieur et de la recherche (CPU, CDEFI, CNRS, Inserm, Inra, Inria, Cnous) ainsi que le ministère de l'Intérieur et celui de l'Europe et des Affaires étrangères. La convention entre le Collège de France et le ministère de l'Enseignement supérieur et de la Recherche portant sur le programme PAUSE a été approuvée par l'assemblée du Collège de France du 26 mars 2017.

Au terme d'un processus d'évaluation, le programme soutient par des financements incitatifs les établissements français d'enseignement supérieur et des organismes de recherche qui s'engagent à recruter les scientifiques en danger et à les accompagner dans leur insertion professionnelle et personnelle.

Au-delà du devoir éthique face à des situations individuelles souvent dramatiques, la solidarité avec les scientifiques étrangers en situation d'urgence constitue une ressource de savoirs d'un intérêt majeur pour le monde académique et la recherche scientifique et, au-delà, la société dans son ensemble.

Le programme Pause a vocation à se développer grâce à de nombreux partenariats publics comme privés. À cette fin, un fonds de souscription pour le programme a été ouvert à la Fondation de France.

\section{LEGS}

Les ressources mises à la disposition du Collège par divers donateurs lui permettent, chaque année, de soutenir sa politique scientifique. Pour un historique des legs, voir Annuaire du Collège de France 2014-2015, Paris, Collège de France, 2016, p. 27-34.

\section{Singer-Polignac}

Le Collège de France a reçu depuis 1950, en souvenir de Winnaretta SINGER, princesse Edmond de Polignac, des dons importants dont les revenus doivent servir, de façon générale, « au progrès des connaissances ». L'affectation précise des fonds est déterminée chaque année par l'assemblée des professeurs.

\section{Gustave Schlumberger}

Par décret du 24 juin 1932, l'administrateur du Collège de France a été autorisé à accepter les legs faits au Collège par Gustave SCHLUMBERGER. Les revenus des sommes provenant de ces legs doivent être affectés d'une part à des études d'histoire et d'archéologie byzantines, d'autre part à des études de numismatique. 


\section{Jean Ébersolt}

Madame Jean ÉBERSOLT a fait une donation en 1968 dont les arrérages doivent être affectés au développement des études d'Histoire et civilisation de Byzance.

\section{Voronoff}

Par décret du 28 septembre 1920, l'administrateur du Collège de France a été autorisé à accepter, au nom du Collège, la donation faite à cet établissement par madame Frances Évelyn BosTwICK, épouse VORONOFF, pour la création et l'entretien d'une "Station de chirurgie expérimentale, fondation Voronoff ».

Depuis le décès de la donatrice, et selon sa volonté, le revenu annuel est attribué aux laboratoires de biologie, d'histologie ou autres laboratoires similaires, selon l'avis de l'Assemblée des professeurs.

\section{Paul Delheim}

Par décret du 5 juin 1956, l'administrateur du Collège de France a été autorisé à accepter le legs fait au Collège de France par J. DELLHEIM «pour aider les jeunes savants peu fortunés qui, après avoir fait de fortes études, seront jugés aptes à poursuivre leurs travaux dans les laboratoires du Collège de France, en vue de recherches scientifiques susceptibles de contribuer au soulagement de l'humanité ». Le prix Dellheim pour l'année 2004 est attribué à M. FAZILLEAU, pour 2005 à M. LIYUAN et pour 2006 à Mariano SIGMAN. Le prix Delheim est attribué en 2007 à Stéphane ROMERO, en 2010 à Franck LEBRIN et à Raoul TORERO-IBAD, en 2011 à Thomas MATIVET, en 2013 à Elphège-Pierre NORA, en 2016 à Hocine REKAÏK, en 2017 à Hsin Chieh WU et en 2018 à Pascale VONAESCH.

\section{Antoine Lacassagne}

Par décret du 6 janvier 1964, l'administrateur du Collège de France a été autorisé à accepter le don fait au Collège de France par Antoine LACASSAGNE, qui fut titulaire de la chaire de Médecine expérimentale (de 1951 à 1954), du montant du prix de 10000 dollars que lui a décerné l'Organisation des Nations unies pour ses travaux sur le cancer.

Les revenus de cette somme ont permis d'inviter de jeunes biologistes français ou étrangers à venir chaque année au Collège exposer, en français, les résultats de leurs recherches.

Des conférences ont été données en 1966, par le Docteur J. HAREL; en 1967, par François GROS; en 1968, par le Docteur Patrick DEROME; en 1970, par Georges N. COHEN ; en 1971, par le Docteur Michel BoIRON ; en 1972, par François CHAPEVILLE ; en 1973, par Nicole Le DoUARIN ; en 1974, par Claude Kordon ; en 1975, par Luc MONTAGNIER ; en 1976, par Jean- Paul LÉVY ; en 1977, par Robert FAUVE.

L'Assemblée des professeurs du 20 février 1977 a décidé que désormais serait attribué un prix Antoine Lacassagne auquel seraient associées deux conférences ; son montant est de 1600 euros. La demande a été faite et approuvée en assemblée (mars 2009) de décerner le prix tous les ans. Depuis 2013, le montant du prix est fixé à 6000 euros. 
Prix décerné en 1977 à Andrée TIXIER-VIDAL, en 1978 à Serge JARD, en 1979 à Michel IMBERT, en 1980 à Jacques GLOWINSKI, en 1981 à Guy BLAUDIN DE THÉ, en 1982 à Jean GIRARD, en 1983 à Pierre FREYCHET, en 1984 à François CUZIN, en 1985 à Michel HAMON, en 1986 à Dominique STEHELIN, en 1987 à Emmanuel PIERROT-DESEILlignY, en 1988 à Pierre TiOllais, en 1989 à Jean-François NICOLAS, en 1990 à Philippe KouRILSKY, en 1991 à Françoise DIETERLEN, en 1992 à Anne-Marie THIERry, en 1993 à Gérard COUlY, en 1994 à Alain DOUCET, en 1995 à Antonio CoutinHO, en 1996 à Jean-Antoine GIRAUD, en 1997 à Alexeï GRANTYN, en 1998 à Marie-Aimée TEILLET, en 1999 à Jules-Alphonse HofFMANN, en 2002 à Werner GRAF, en 2004 à Michel BORNENS, en 2005 à Ana CUMANO, en 2006 à Philippo RIJLI, en 2007 à Thomas BOURGERON, en 2011 à Clotilde THÉRY, en 2013 à Sonia GAREL, en 2016 à Hélène Monique PUCCIO, en 2017 à Virginie ORGOGOZO.

\section{Antoine Meillet}

Par décret du $1^{\text {er }}$ septembre 1937, l'administrateur du Collège de France a été autorisé à accepter le legs fait au Collège par Antoine MEILLET, qui fut titulaire de la chaire de Grammaire comparée (de 1906 à 1936). Les revenus de ce legs sont affectés à des études de linguistique théorique, suivant les vœux du donateur.

\section{Claude-Antoine Peccot}

Plusieurs donations successives (en 1886, en 1894, en 1897, en 1902) ont permis de créer d'abord des bourses, transformées par la suite en prix ; puis, en outre, à partir de 1900, une charge de cours, au bénéfice de mathématiciens âgés de moins de trente ans et s'étant signalés dans l'ordre des mathématiques théoriques ou appliquées.

Voici, depuis l'origine, la liste des personnes qui ont bénéficié des prix ou ont reçu la charge du cours :

Chargés de cours

\begin{tabular}{|c|c|c|c|}
\hline 1900 & Émile BOREL & 1912 & Jean CHAZY \\
\hline 1901 & Émile BOREL & & Albert CHATELET \\
\hline 1902 & Émile BOREL & 1913 & Arnaud DENJOY \\
\hline 1903 & Henri LEBESGUE & 1914 & Édouard-René GARNIER \\
\hline 1904 & René BAIRE & & Maurice GEVREY \\
\hline 1905 & Henri LEBESGUE & 1915 & Édouard-René GARNIER \\
\hline 1906 & Guillaume SERVANT & 1918 & Gaston JULIA \\
\hline 1907 & Pierre Boutroux & 1919 & Georges GIRAUD \\
\hline 1908 & Pierre Boutroux & & Paul LÉVY \\
\hline 1909 & Ludovic ZORETTI & 1920 & Léon BRILLOUIN \\
\hline 1910 & Émile TRAYNARD & & Gaston JULIA \\
\hline 1911 & Louis RÉMY & 1921 & Maurice JANET \\
\hline
\end{tabular}


1922 René THIRY

1923 Robert DELTHEIL

Torsten CARLEMAN

1924 René LAGRANGE

1925 Marcel LEGAUT

1926 Henri MillouX

1927 Joseph KAMPÉ DEFÉRIET

Yves ROCARD

1928 Szolem MANDELBROJT

1929 Jean FAVARD

1930 Wladimir BERNSTEIN

1931 Jean DELSARTE

1932 Henri CARTAN

André WEIL

1933 Jean DIEUdONNÉ

Paul DUBREIL

1934 René De Possel

Jean LERAY

1935 Marie-Louise

DUBREIL-JACOTIN

1936 Georges BouRION

Jean-Louis DESTOUCHES

1937 Jacques SOLOMON

Claude CHEVAlLeY

1938 Frédéric MARTY

1941 Claude CHABAUTY

1942 Gérard PÉTIAU

1943 Jean VILLE

Marie-Antoinette TonNELAT

1944 Hubert DELANGE

Jacques DUFRESNOY

1945 André LICHNEROWICZ

1946 Laurent SCHWARTZ

Jacqueline FERRAND

1947 Gustave CHOQUET

1949 Roger APÉRY

1950 Jacques DENY

1951 Jean-Louis KoszUL

Evry SCHATZMAN
1952

1953

195

1955

1956

1957

1958

1959

1960

1961

1962

1963

1964

1965

1966

1967

1968 Uriel FRISCH

Jean GINIBRE

Pierre GRISVARD

1969

Yves MEYER

Claude MORLET

Michel RAYNAUd

1970

Gabriel MoKoBODZKI

Roger TEMAM

1971

Jean-Pierre FERRIER

Hervé JACQUET

Gérard SCHIFFMANN

1972

Louis BouTET DE MONVEL

Pierre DELIGNE

1973

Jean-Michel BONY

François LAUDENBACH

1974 Haïm BRÉZIS

Michel DUFLO 


\begin{tabular}{|c|c|c|c|}
\hline \multirow{4}{*}{1975} & Jean ZINN-JUSTIN & 1994 & Ricardo PEREZ-MARCO \\
\hline & Jean-Marc FONTAINE & & Frédéric HELEIN \\
\hline & André NEVEU & 1995 & Éric SÉRÉ \\
\hline & Robert ROUSSARIE & & LoÏc MEREL \\
\hline \multirow[t]{2}{*}{1976} & Alain CONNES & 1996 & Laurent LAFFORGUE \\
\hline & Bernard TEISSIER & 1997 & Christophe BREUIL \\
\hline \multirow[t]{2}{*}{1977} & Luc TARTAR & & Christine LESCOP \\
\hline & Michel WALDSCHMIDT & 1998 & Andrei MOROIANU \\
\hline \multirow[t]{2}{*}{1978} & Jean LANNES & 1999 & Philippe MiCHEL \\
\hline & Arnauld BEAUVILLE & & Wendelin WERNER \\
\hline \multirow[t]{2}{*}{1979} & Bernard GAVEAU & 2000 & Emmanuel GRENIER \\
\hline & Grégory CHOODNOVSKY & & Raphaël RoUQUIER \\
\hline 1980 & Gilles ROBERT & 2001 & Vincent LAFFORGUE \\
\hline \multirow[t]{3}{*}{1981} & Michel TALAGRAND & & Frédéric LEROUX \\
\hline & Gilles PISIER & 2002 & Denis Auroux \\
\hline & Christophe SOULÉ & & Thierry BODINEAU \\
\hline \multirow[t]{2}{*}{1982} & Jean-Bernard BAILLON & 2003 & Franck BARTHE \\
\hline & Jean-Luc BRYLINSKI & & Cédric VILLANI \\
\hline 1983 & Jean-Loup WALDSPURGER & 2004 & Laurent FARGUES \\
\hline \multirow[t]{3}{*}{1984} & Pierre-Louis LIONS & & Laure SAINT-RAYMOND \\
\hline & Guy HENNIART & 2005 & Artur AvILA \\
\hline & Laurent CLOZEL & & Stefaan VAES \\
\hline 1985 & Joseph OESTERLÉ & 2006 & Laurent BERGER \\
\hline 1986 & Jean-Pierre DEMAILLY & & Emmanuel BREUILLARD \\
\hline \multirow[t]{2}{*}{1987} & Jean-Michel CORON & 2007 & Erwan RoUSSEAU \\
\hline & Jean-Christophe YoCCOZ & & Jérémie SzEFTEL \\
\hline \multirow[t]{2}{*}{1988} & Jean-Lin JOURNÉ & 2008 & Karine BEAUCHARD \\
\hline & Jean-Claude SIKORAV & & Gaëtan CHENEVIER \\
\hline \multirow[t]{2}{*}{1989} & Bernard LARROUTUROU & 2009 & Joseph AYOUB \\
\hline & Jean-François LEGALL & & Julien DUBÉDAT \\
\hline \multirow[t]{2}{*}{1990} & Jean-Benoît BosT & 2010 & Antoine TouZÉ \\
\hline & Benoît PERTHAME & 2011 & Sylvain ARLOT \\
\hline \multirow[t]{2}{*}{1991} & Olivier MATHIEU & & Anne-Laure DALIBARD \\
\hline & Claude Viterbo & 2012 & Alessio FIGALLI \\
\hline \multirow[t]{3}{*}{1992} & Claire VoIsIN & & Vincent PILLONI \\
\hline & Fabrice BETHUEL & 2013 & Valentin FERAY \\
\hline & Noam ELKIES & & Christophe GARBAN \\
\hline \multirow[t]{2}{*}{1993} & Marc Rosso & & Peter SCHOLZE \\
\hline & François GOLSE & 2014 & François CHARLES \\
\hline
\end{tabular}




\begin{tabular}{|c|c|c|c|}
\hline & Nicolas RougERIE & 2017 & Raphaël BEUZART-PLESSIS \\
\hline \multirow[t]{2}{*}{2015} & Gabriel DosPINESCU & & Olivier TAÏ̈I \\
\hline & Hugo DUMINIL-COPIN & 2018 & Yannick BONTHONNEAU \\
\hline \multirow[t]{2}{*}{2016} & Nicolas CURIEN & & Camille HORBEZ \\
\hline & Marco RoBALO & 2019 & Jacek JENDREJ \\
\hline
\end{tabular}

\section{Prix Peccot-Vimont}

À partir de 1885 : L. Bortniker, Jacques HADAMARD, Élie CARTAN, Jules BOCQUET, Jules DRACH, Louis-Emmanuel LEROY, Adolphe BÜHL, Gabriel MESURET, Pierre FATOU, René-Maurice FréChet, Henri Galbrun, Osée Marcus, Jean Chazy, Albert LABORDE-ScAR, Paul Frion, Gabriel PÉlissier, René GARNIER, Emmanuel FAUré-Fremiet, Émile TerroIne, RouX, Maurice GEVREY, F. LAFOrE, Joseph MARTY, Georges GIRAUd, Maurice JANET, Coty, Paul LÉVY, Gaston JULIA, Léon Brillouin, Marcel Courtines, Szolem MAndelbrojt, Yves RocARD, Wladimir BERnsteIn, Henri CARTAN, André WeIL, Jean DiEUdonnÉ, Paul DubreIL, René DE Possel, Jean LeRAY, Georges BouRION, Jean-Louis DESTOUCHES, Jacques Solomon, Claude Chevalley, Frédéric Roger, Daniel Dugué, Gérard Petiau, Hubert DELANGE, Jacques Dufresnoy, Laurent SCHWARTZ, Jacqueline FerRAND, Roger APÉRY, Jacques DENY, Jean-Louis KosZul, Jean COMBES, Jean-Pierre SERRE, Paul Malliavin, Maurice Roseau, Bernard Malgrange, François BruHat, Pierre CARTIER, Paul-André MeYer, Marcel Froissart, Michel DemaZure, Gabriel MOKOBODZKI, Hervé JACQUET, Haïm BRÉZIS, Alain CONNES, Grégory Choodnovsky, Jean-Pierre Demailly, Jean-Benoît Bost, Noam Elkies, Laurent LAFForgue, Philippe Michel, Vincent LAFForgue, Cédric Villani, Gaëtan ChEnEVIER, Alessio Figalli, Peter SchOlze, François Charles.

\section{Loubat}

Par deux décrets en date du 16 avril 1902 et 28 juillet 1903, le Collège de France a été autorisé à accepter la donation faite par le duc de LOUBAT, membre associé de l'Institut de France, en vue de la fondation, dans l'établissement, d'un cours complémentaire d'Antiquités américaines.

Ce cours a été confié à Léon LEJEAL (1902-1907), puis au docteur Louis CAPITAN (1908-1929). Depuis 1939, les revenus de cette fondation ont permis de demander des conférences à des américanistes.

Liste des conférenciers invités par le Collège de France :

$\begin{array}{llll}1939 & \text { Jacques SOUSTELLE } & 1950 & \text { Claude LÉVI-STRAUSS } \\ 1941 & \text { André LEROI-GOURHAN } & 1952 & \text { Paul RIVET } \\ 1942 & \text { Raoul D’HARCOURT } & 1953 & \text { Alfred MÉTRAUX } \\ 1943 & \text { Maurice LEENHARDT } & 1955 & \text { Jehan VELLARD } \\ 1945 & \text { Marcel GIRAUD } & 1965 & \text { Pierre CLASTRES } \\ 1946 & \text { Henri VALLOIS } & 1969 & \text { Frédéric ENGEL } \\ 1948 & \text { Guy STRESSER-PÉAN } & 1973 & \text { Annette LAMING-EMPERAIRE }\end{array}$




$\begin{array}{llll}1974 & \text { Jean-Claude QUILICI } & 1996 & \text { Philippe DESCOLA } \\ 1975 & \text { Georges LARROUY } & 1997 & \text { Carmen BERNAND } \\ 1977 & \text { Nathan WACHTEL } & 1998 & \text { Jacques GALINIER } \\ 1980 & \text { Christian DUVERGER } & 2002 & \text { Danielle LAVALLÉE } \\ 1995 & \text { Serge GRUZINSKI } & 2004 & \text { Jon LANDABURU }\end{array}$

\section{Michonis}

Par décret du 10 mars 1903, G. Michonis a légué au Collège de France une somme dont les revenus doivent servir à «faire faire, toutes les fois que ce sera possible, par un savant ou un penseur étranger désigné par les professeurs ou l'administrateur du Collège de France, et qui sera, autant que les circonstances le permettront, au moins une fois sur trois un philosophe ou un historien de sciences religieuses, une série de conférences ». L'exécution des volontés de G. MicHONIS a commencé en 1905.

Liste des conférenciers invités par le Collège :

\begin{tabular}{|c|c|c|c|}
\hline \multirow[t]{2}{*}{1905} & Édouard NAVILLE & 1926 & Nicolas ALEXEIEFF \\
\hline & Franz CUMONT & 1927 & Ernest MURET \\
\hline 1906 & Guglielmo FERRERO & 1929 & Wolfgang KOEHLER \\
\hline \multirow[t]{2}{*}{1908} & Charles MiCHEL & 1930 & Georges D. BIRKOFF \\
\hline & Alexandru XÉNOPOL & 1933 & Magnus OLSEN \\
\hline \multirow[t]{2}{*}{1910} & Christophe NYROP & 1934 & Harl JABERG \\
\hline & Édouard MONTET & & Jacob JUD \\
\hline \multirow[t]{2}{*}{1912} & LORENTZ & 1935 & Stanislas Kот \\
\hline & GOMPERZ & 1936 & Jacques PIRENNE \\
\hline 1914 & Maxime KOWALEWSKY & 1937 & Albert МiсHOTTE \\
\hline \multirow[t]{3}{*}{1915} & Georges DOUTREPONT & 1938 & Giorgio LEVI DELLA VIDA \\
\hline & Paul DELANNOY & 1940 & HROZNY \\
\hline & Albert BRACHET & 1942 & Jean PIAGET \\
\hline \multirow[t]{2}{*}{1916} & Charles DE LA VALLÉE- & 1943 & Franz CUMONT \\
\hline & POUSSIN & 1945 & Alexandre RosetTI \\
\hline 1917 & Joséphine IOTEYKO & 1947 & Georges Dossin \\
\hline 1918 & Paul FRÉDÉRICQ & & Hans SELYE \\
\hline 1919 & Henri PIRENNE-ANESAKI & 1948 & Charles DETOLNAY \\
\hline \multirow[t]{2}{*}{1920} & Michel RostovtzeFF & 1951 & Étienne LAMOTTE \\
\hline & JORGA & 1956 & Gino LuZZATto \\
\hline 1922 & Albert EINSTEIN & & Walter E. PETRASCHECK \\
\hline 1923 & Raffaele ALTAMIRA & 1961 & Théodor W. ADORNO \\
\hline 1924 & Ettore PAIS & 1965 & V.I. ABAEV \\
\hline 1925 & Holger PEDERSEN & 1968 & Théodor W. ADORNO \\
\hline
\end{tabular}




$\begin{array}{llll}1973 & \text { Ludovico GEYMONAT } & 2007 & \text { Philippe BORGEAUD } \\ 1984 & \text { Jean RUDHART } & 2010 & \text { Simon PRICE } \\ 1989 & \text { David WigGins } & 2012 & \text { Glen BowERSOCK }\end{array}$

\section{Saintour}

Par décret du 25 juillet 1889, l'administrateur du Collège de France a été autorisé à accepter le legs fait au Collège de France par le $\mathrm{D}^{\mathrm{r}}$ SAINTOUR pour la fondation d'un prix. Ce prix, périodiquement revalorisé, est décerné tous les deux ans par l'assemblée des professeurs, sur la présentation qui lui est faite, $d$ 'après un roulement déterminé, par l'une des trois sections instituées à cet effet. Chaque section regroupe les titulaires de chaire pour lesquels les différentes Académies composant l'Institut ont concurremment avec le Collège le droit de présentation. Les trois sections comprennent ensemble la totalité des professeurs.

Ce prix a été attribué depuis sa fondation (1893) à M. MATIGNON, M. ChassinoIT, Abel Lefranc, Philippe Glangeaud, M. Laurent, M. Chauvin, M. Hallion, M. Lenestour, M. Lacôte, Ernest Charles, Léon Lecornu, M. Homo, Jules Chauvin, Paul Langevin, Gaston Colin, Gaston Cohen, Pierre Leroux, Georges MaYer, Alexandre Dufour, Alfred Ernout, Louis Bodin, Paul MAZon, René Henry, Julien Barat, $M^{\text {lle }}$ Chevroton, $M^{\text {lle }}$ Loyez, M. Delaruelle, M. VAlois, M. CHABOt, Édouard SAlles, M. CopauX, Claude Blanchel, Jules Bloch, M. BoudréauX, M. GafFiot, M. Virolleaud, M. Brillaut, Henri Clouzot, Georges LeCArpentier, Achille Millien, M. Champy, M. LerouX, M. LÉvy, M. et $\mathrm{M}^{\text {me }}$ Marouseau, M. Lejeune, M. Terroine, M. RouX, $\mathrm{M}^{\mathrm{me}}$ BoudréauX, M. Ouveriaux, M. Foulet, M ${ }^{\text {lle }}$ Blanchard Demonge, M. Boulard, Bernard Leroy, André PÉZARD, M. RANDouin, M Mle IOTEYKo, Prosper Alfaric, M. Doutrepont, Mlle Maitret, M. Delapparent, Alfred Quinquaud, M. Meyerson, Henri Heine, Taha Hussein, André VaILlant, M. MarTy, l'abbé Busson, M. Sartre, M. FréJacques, Émile Benveniste, M. Sommerfelt, M. CARidroit, M. Misconi, M. BAudot, Albert Houstin, Marie Cochet, M. Millon, $M^{\text {lle }}$ Marchal, M ${ }^{\text {lle }}$ Bezard, M. Contineau, M. Umbegaun, M. P. Noailhon, Boris Ephrussi, M. Casteras, M. Salovine, M. Samara, M. Martini, Paul Émard, Alice Hulubet, Robert Courrier, Jean Filliozat, Pierre PASCAL, Marc CoHn, Maurice LEENHARDT, Étienne WolfF, M ${ }^{\text {me }}$ D'Also, M. Chapire, M. Rolland, M. Biquard, Jean Fourquet, André Chevalier, Albert Dauzat, René Clozier, Jules Driessens, René Pintard, M. KLein, René Vallois, Charles Morazé, M. A. Jost, Marcel Simon, M. Lemagnen, Paul-Henri Michel, André AdAM, M ${ }^{\text {me }}$ SKreb-Guilcher, Rév. Père Estugière, Yves LeCorre, M. Adigard des Gautries, Pierre RanCASTel, Armand Hampé, Jacques Fontaine, Roger Guillemin, Louis-Charles Damais, Jean PouillouX, Guy Lasserre, Paul Kessler, Paul Garelli, Martha SPItZer, Henri Rolland, Paul Pelissier, André Landesman, Jean Riché, M. W. StreifF, Valentin NikIPROWETZKY, Richard GASCON, Gilles Granger, Hans GlatTli, Odette TAFFAnel, Michel Gaudin, Hervé Savon, Victor Goldschmidt, Neil Sullivan, Venceslas Kruta, M. M. Flato, M. R. TurCan, Daniel EstèVe, Jean-Pierre MahÉ, André Langaney, Jean-Marie Durand, Marianne MAHN-Lot, Loïc Merel, Willy Clarysse, Claude GhesquiÈre, Pierre Milza, M. Piemontese, Christian Giaume. 